\title{
OPTIMAL $A$ PRIORI ERROR ESTIMATES IN WEIGHTED SOBOLEV SPACES FOR THE POISSON PROBLEM WITH SINGULAR SOURCES
}

\author{
IGNACIO OJEA*
}

\begin{abstract}
We study the problem $-\Delta u=f$, where $f$ has a point-singularity. In particular, we are interested in $f=\delta_{x_{0}}$, a Dirac delta with support in $x_{0}$, but singularities of the form $f \sim\left|x-x_{0}\right|^{-s}$ are also considered. We prove the stability of the Galerkin projection on graded meshes in weighted spaces, with weights given by powers of the distance to $x_{0}$. We also recover optimal rates of convergence for the finite element method on these graded meshes. Our approach is general and holds both in two and three dimensions. Numerical experiments are shown that verify our results, and lead to interesting observations.
\end{abstract}

Mathematics Subject Classification. 35J05, 65N30, 65N12, 65N15, 65 Y20.

Received August 14, 2019. Accepted September 1, 2020.

\section{INTRODUCTION}

The goal of this paper is to apply the finite element method for solving the Poisson equation with sources that present a point singularity. In particular, we study:

$$
\begin{cases}-\Delta u(x)=f(x) & x \in \Omega \subset \mathbb{R}^{n} \\ u(x)=0 & x \in \partial \Omega\end{cases}
$$

where $f$ is a measure with a singularity in an interior point $x_{0} \in \Omega$. This singularity is characterized by the fact that $f$ does not belong to $H^{-1}(\Omega)$, but is a bounded operator $f: H_{0,-\alpha}^{1}(\Omega) \rightarrow \mathbb{R}$, where $H_{0,-\alpha}^{1}(\Omega)$ is the Sobolev weighted space, with weight $\left|x-x_{0}\right|^{-2 \alpha}$, for certain values of $\alpha>0$. Our model case is $f=\delta_{x_{0}}$, a Dirac delta distribution supported on $x_{0} \in \Omega$, such that:

$$
\int_{\Omega} \varphi(x) \delta_{x_{0}}(x) \mathrm{d} x=\varphi\left(x_{0}\right), \quad \forall \varphi \in C^{1}(\bar{\Omega}) .
$$

However, other functions can be considered. For example: if $f$ is such that $f(x) \sim\left|x-x_{0}\right|^{-s}$ near $x_{0}$, our approach can be applied to obtain a weighted setting for the problem, as well as optimal rates of convergence for the finite element method (see Example 2.4).

Throught the paper, $\Omega$ is a convex polygon or polyhedron or a (not necessarily convex) smooth domain.

Keywords and phrases. Weighted Sobolev spaces, a priori error estimates, finite elements.

Departamento de Matemática Facultad de Ciencias Exactas y Naturales, Universidad de Buenos Aires, Buenos Aires, Argentina.

* Corresponding author: iojea@dm.uba.ar 
Problem (1.1) with $f=\delta_{x_{0}}$ has been largely studied. Since the right hand side does not belong to $H^{-1}(\Omega)$, a non-standard approach is needed for setting the continuous problem, as well as for obtaining optimal orders of convergence for the numerical solution given by the finite element method. Indeed, in [8], the author studied elliptic problems with right hand side given by a Borel measure, and showed that the solution belongs to the Sobolev space $W^{1, p}$ with $1 \leq p<\frac{n}{n-1}$. Moreover, an error analysis is provided, showing that the error of the finite element solution on quasi-uniform meshes of size $\sim h$, using functions that are locally polynomials of degree $\leq 1$, is of order $h$ for $n=2$, and of order $h^{\frac{1}{2}}$ for $n=3$, whereas it is well known that when the solution is regular enough (it belongs to $H^{2}(\Omega)$ ) the error is of order $h^{2}$, with indepenpendence of the dimension. Other results are obtained with different approaches. For example, an a priori error analysis is carried out in [6,28], where the error is measured in $L^{2}$ and in fractional Sobolev spaces $H^{s}$ for some $0<s<1$. In [4], the two dimensional problem is considered and weighted Sobolev spaces are used for obtaining estimates for the error in $L^{2}$ on graded meshes. A weighted approach is also performed in [24], where a general piecewise polynomial approximation theory in weighted spaces is developed for general shape-regular meshes and weights in $A_{p}$. Problem (1.1) with $f=\delta_{x_{0}}$ is studied as an application, obtaining an $L^{2}$ error estimate on quasi-uniform meshes, in terms of a weighted norm of $\nabla u$. In [20], quasi-uniform meshes are considered, obtaining quasi-optimal rates of convergence for finite element methods of order one, and optimal rates of convergence for higher order methods. However, the authors only consider local error norms: the error is measured on a domain excluding the singularity. On the other hand, an a posteriori error analysis is performed in [5] (for the 2D problem only) giving estimates in $L^{p}$, with $1<p<\infty$ and in $W^{1, p}$ for $1<p<2$; and in [1] where weighted norms are used.

Here we are interested in problem (1.1) as a model for heat diffusion produced by the heating of gold nanoparticles through laser beams (see e.g., [26]). Nanoparticles are represented as point-sources, and (1.1) models the steady-state heat equation.

Our goal is to give a general theory for setting the problem in weighted Sobolev spaces and to derive from that setting a priori error estimates on graded meshes that allow us to recover optimal rates of convergence. In that sense, this paper is a continuation of [12]. As it is pointed out in [1], the weighted approach seems to be more appropriate than $W^{1, p}$ spaces (used in [5]) or $H^{s}$ spaces (considered in [6,28]), since the norm of the weighted spaces is only weakened near the singularity, and not in the whole domain $\Omega$. However, as far as we are aware of, this fact had not yet been exploited to give a comprehensive theory that leads to optimal rates of convergence for the finite element method.

Our weighted setting is based on the ideas of [10]. Consider $d(x)=\left|x-x_{0}\right|$, we define: $L_{\alpha}^{2}(\Omega)$ the weighted space equipped with the norm:

$$
\|v\|_{L_{\alpha}^{2}(\Omega)}:=\left(\int_{\Omega} v(x)^{2} d(x)^{2 \alpha} \mathrm{d} x\right)^{\frac{1}{2}},
$$

and $H_{\alpha}^{1}(\Omega)$ the space of functions in $L_{\alpha}^{2}$ with derivatives in $L_{\alpha}^{2}$. Our weak formulation takes $H_{\alpha}^{1}$ as the ansatz space and $H_{-\alpha}^{1}$ as the test space. For these spaces, a well posedness result is needed. Such a result is given in a very general setting in [10]. Regretfully, the proof there provided is flawed due to a mistake that affects both the continuous and the discrete cases. Satisfactory proofs for the continuous problem can be found in $[12,25]$ (see Thm. 2.1 and the discussion below for details).

On the other hand, to obtain discrete stability results in weighted norms is a rather difficult task. In fact, thanks to an extrapolation theorem due to Rubio de Francia, stability in $H_{\omega}^{1}$ for every $\omega \in A_{1}$ would imply stability in $W^{1, p}$ for every $2<p<\infty$. A particularly tough obstacle that arises in the discrete case is that the arguments usually applied are mesh-dependent. For example: the classical max-norm analysis (see e.g., [7], Chap. 8) can be taken advantage of for studying weighted norms, but this holds only for quasi-uniform meshes, that give sub-optimal error estimates for singular problems such as ours. Moreover, the arguments in Chapter 8 of [7] are based on the assumption of $W^{2, p}$ regularity for some $p>n$. This hypothesis is verified for every convex polygon, but in the case of polyhedra, it requires the artificial condition that the inner dihedral angles be smaller than $\frac{3 \pi}{4}$ (see [17] and [22], Thm. 7.1). In [17] a max-norm estimate is proven for convex polyhedra, avoiding this unnecessary restriction. The ideas used there were adapted in [12] for proving a weighted stability result 
for general weights, but only for quasi-uniform meshes. Here we apply a similar approach for proving weighted stability for graded meshes. This result relies on the weighted setting established in [12]. Our technique resembles the one used in [21], where the author proves $W^{1, p}$ stability for convex polygons on meshes graded towards the vertices of the domain.

It is important to notice that both [1] and the results given for a Dirac delta right hand side in [24] depended on the well posedness result given in [10]. In particular, the a priori estimates in [24] depended also on the stability of the Galerkin projection that is stated in a very general context in [10], but is affected by the same mistake that invalidates the well posedeness result. Both these issues are fixed in [12], in the discrete case for quasi-uniform meshes. It is easy to check that the weight considered in [24] is included in the restricted class of $A_{2}$ weights for which the stability of the Galerkin projection is proven in [12].

Once the stability of the Galerkin projection is established, we proceed to prove error estimates on graded meshes, that recover optimal rates of convergence provided that the graduation parameter satisfies certain restrictions. In the particular case $f=\delta_{x_{0}}, n=2$ and $L^{2}$ estimates, our results generalize the one obtained in [4]. In that paper, a max-norm argument is used which worsens the error estimate by a logarithmic factor. Indeed, the result of [4] is:

$$
\left\|u-u_{h}\right\|_{L^{2}(\Omega)} \leq C \log (h)^{\frac{3}{2}} h^{2} .
$$

However, it is important to remark that our results hold in both 2 and 3 -dimensional problems and that the weighted setting allows us to obtain error estimates for $u-u_{h}$ and its derivatives, in weighted spaces where the solution $u$ naturally belongs.

The paper is organized as follows: in Section 2 we establish the notation and present some auxiliary results. In Section 3 we give a weighted a priori estimate, needed in Section 4, where we study the stability of the discrete solution on meshes graded towards $x_{0}$. In Section 5 we give a priori error estimates in $H_{\alpha}^{1}$ and in $L_{\beta}^{2}$, both for $n=2$ and $n=3$. Finally, Section 6 shows numerical experiments that agree with the theoretical predictions.

\section{Preliminaries}

We consider $\Omega \subset \mathbb{R}^{n}$ for $n=2$ or $n=3$ a bounded domain. Certain assumptions should be made on $\Omega$ for our results to hold (see Rem. 3.4). In particular, we can take $\Omega$ a convex polygon or polyhedron, or a domain of class $\mathcal{C}^{1,1}$ (not necessarily convex). By $\mathcal{C}^{1,1}$ we mean that $\partial \Omega$ can be locally described by a function $\psi$ such that $\psi$ is $C^{1}$ and the derivatives of both $\psi$ and $\psi^{-1}$ are Lipschitz.

For $\omega: \Omega \rightarrow \mathbb{R}_{\geq 0}$, we define $L_{\omega}^{p}(\Omega)$ the space of functions $v$ such that: $\int_{\Omega}|v|^{p} \omega \mathrm{d} x<\infty$. Accordingly, we have $W_{\omega}^{1, p}(\Omega)$, the weighted Sobolev space formed by all functions $v$ such that $v$ and its derivatives of first order belong to $L_{\omega}^{p}(\Omega)$. As usual $W_{0, \omega}^{1, p}(\Omega)=\overline{C_{0}^{\infty}}$. For $p=2$ we write $H_{\omega}^{1}$ and $H_{0, \omega}^{1}$. $\omega$ is said to be in the class $A_{p}$ of Muckenhoupt for $1<p<\infty$, if:

$$
[\omega]_{A_{p}}=\sup _{Q}\left(\frac{1}{|Q|} \int_{Q} \omega\right)\left(\frac{1}{|Q|} \int_{Q} \omega^{-\frac{1}{p-1}}\right)^{p-1}<\infty,
$$

where the supremum is taken over all cubes $Q$. On the other hand, $\omega$ is in $A_{1}$ if:

$$
[\omega]_{A_{1}}=\sup _{x} \frac{\mathcal{M} \omega(x)}{\omega(x)}<\infty
$$

where $\mathcal{M} g(x)=\sup _{Q \ni x} \frac{1}{|Q|} \int_{Q} g(y) \mathrm{d} y$ is the Hardy-Littlewood maximal operator. It is known (see [14]), that for $1 \leq p<\infty$, a weight of the form $\omega(x)=d(x)^{\beta}$ belongs to $A_{p}$ if:

$$
-n<\beta<n(p-1) .
$$

For any exponent $1<p<\infty$ we denote $p^{\prime}$ such that $\frac{1}{p}+\frac{1}{p^{\prime}}=1$. Through the paper, $C$ stands for a constant that may change from line to line. When necessary, we point out how $C$ depends on the parameters. Finally, we 
say that two magnitudes $a$ and $b$ are equivalent, and write $a \sim b$ when there are constants $C_{1}, C_{2}$ independents of $a$ and $b$ such that $C_{1} a \leq b \leq C_{2} b$. This makes sense particularly when, given a set of numbers $\mathcal{A}$ we say $a \sim b$ for every $a \in \mathcal{A}$, which means that $C_{1}$ and $C_{2}$ are the same for every $a$.

Furthtermore, let us define $A_{p}(\Omega) \subset A_{p}$ a subclass of weights such that for every $\omega \in A_{p}(\Omega)$ there is some $\varepsilon>0$, a neighbourhood $G$ of $\partial \Omega$ and a constant $\omega_{L}$ such that:

$-\{x \in \Omega: \operatorname{dist}(x, \partial \Omega)<\varepsilon\} \subset G$,

- $\omega$ is continuous on $G$,

- $\omega(x)>\omega_{L} \quad \forall x \in G$.

Our analysis relies on the weighted a priori estimate:

Theorem 2.1. Problem (1.1) with $f \in\left(W_{0, \omega^{-1 /(p-1)}}^{1, p^{\prime}}(\Omega)\right)^{\prime}$, admits a unique solution $u \in W_{0, \omega}^{1, p}(\Omega)$, satisfying:

$$
\left.\|u\|_{W_{0, \omega}^{1, p}(\Omega)} \leq C\|f\|_{\left(W_{0, \omega}^{1, p^{\prime}},(p-1)\right.}(\Omega)\right)^{\prime}
$$

This result is proven in Corollary 2.6 of [12] for $\Omega$ a convex polygon, polyhedron or smooth domain, for every $\omega \in A_{p}$, and $1<p<\infty$; also, in Theorem 4 of [25] for $\Omega$ a Lipschitz domain, for every $\omega \in A_{p}(\Omega)$, and $p$ restricted to a certain interval containing $p=2$. Hence: the extension to a wider class of domains is obtained in exchange for a restriction in the class of weights and in the range for $p$.

We take $p=2$ and $\omega=d^{2 \alpha}$. Following [10], we denote $L_{\alpha}^{2}, H_{\alpha}^{1}, H_{0, \alpha}^{1}$ the spaces with weight $\omega=d^{2 \alpha}$. Applying (2.2) we obtain that $d(x)^{2 \alpha} \in A_{2}$ if $-\frac{n}{2}<\alpha<\frac{n}{2}$. In fact, since $x_{0} \in \Omega, d^{2 \alpha} \in A_{2}(\Omega)$. We write:

$$
(u, v)=\int_{\Omega} u v
$$

the duality pairing for $u \in L_{\alpha}^{2}$ and $v \in L_{-\alpha}^{2}$.

In this context, we set our model problem as:

$$
\text { Given } f \in\left(H_{0,-\alpha}^{1}(\Omega)\right)^{\prime} \text {, Find } u \in H_{0, \alpha}^{1}(\Omega) / \quad(\nabla u, \nabla v)=(f, v) \quad \forall v \in H_{0,-\alpha}^{1}(\Omega) .
$$

Theorem 2.1 says that, for any Lipschitz domain, $x_{0} \in \Omega$ and $-\frac{n}{2}<\alpha<\frac{n}{2}$, (2.3) has a unique solution, that satisfies:

$$
\|u\|_{H_{0, \alpha}^{1}(\Omega)} \leq C\|f\|_{\left(H_{0,-\alpha}^{1}\right)^{\prime}} .
$$

Moreover, for weights in $A_{p}$, Poincaré inequality stands. Hence, we can take $\|\nabla u\|_{L_{\alpha}^{2}(\Omega)^{n}}$, as a norm for $H_{0, \alpha}^{1}(\Omega)$. In fact, a more general result holds:

Theorem 2.2. Let $\Omega \in \mathbb{R}^{n}$ be a $\mathcal{C}^{1,1}$ domain or a convex polygon or polyhedron, $1 \leq \tau_{2} \leq \tau_{1}<\infty, \gamma_{1}, \gamma_{2} \in \mathbb{R}$, such that:

$$
\frac{1}{\tau_{1}}+\frac{\gamma_{1}}{n}>0, \quad \frac{1}{\tau_{2}}+\frac{\gamma_{2}}{n}<1, \quad 0 \leq \gamma_{2}-\gamma_{1} \leq 1,
$$

and

$$
\frac{1}{\tau_{1}}+\frac{\gamma_{1}}{n}=\frac{1}{\tau_{2}}+\frac{\gamma_{2}-1}{n}
$$

Then, there is a constant $C_{P}$ such that

$$
\left\||x|^{\gamma_{1}} v\right\|_{L^{\tau_{1}(\Omega)}} \leq C_{P}\left\||x|^{\gamma_{2}} \nabla v\right\|_{L^{\tau_{2}(\Omega)}},
$$

for every $v \in H_{0}^{1}(\Omega)$, and for every $v \in H^{1}(\Omega)$ such that $\int_{\Omega} v=0$. 
Proof. We can apply a well known result by Sawyer and Wheeden (see [27], Thm. 1), where it is stated that for $Q$ a cube and $v$ with vanishing trace in $\partial Q$ or vanishing mean value in $Q$, the inequality:

$$
\left\|w_{1} v\right\|_{L^{\tau_{1}}(Q)} \leq C_{P}\left\|w_{2} \nabla v\right\|_{L^{\tau_{2}}(\Omega)},
$$

holds (for $\tau_{2} \leq \tau_{1}$ ), if there exist some $r>1$ such that:

$$
A_{w_{1}, w_{2}}^{\tau_{1}, \tau_{2}}(r, B)=|B|^{\frac{1}{n}+\frac{1}{\tau_{1}}-\frac{1}{\tau_{2}}}\left(\frac{1}{|B|} \int_{B} w_{1}^{r \tau_{1}}\right)^{\frac{1}{r \tau_{1}}}\left(\frac{1}{|B|} \int_{B} w_{2}^{r \tau_{2}\left(1-\tau_{2}^{\prime}\right)}\right)^{\frac{1}{r \tau_{2}^{\prime}}} \leq C_{r}<\infty
$$

for every ball $B$. The original result is stated in terms of cubes instead of balls, but both statements are equivalent, and balls are more appropriate for our weights. For our domain $\Omega$, if $v \in H_{0}^{1}(\Omega)$, it can be extended by 0 to a cube, and the result can be applied. On the other hand, for the case $\int_{\Omega} v=0$, a slightly improved result holds for John domains (see [11], Thm. 4.1), provided that the weights satisfies a doubling condition.

We take $w_{1}=|x|^{\gamma_{1}}$ and $w_{2}=|x|^{\gamma_{2}}$ and verify that condition (2.5) is fulfilled, proving the Theorem. We consider two cases:

Let $r_{B}$ be the radious of $B$ and $d_{B}$ the distance of $B$ to the origin. Then, if $r_{B} \leq d_{B}$, we have that $d_{B} \leq|x| \leq 3 d_{B}$. Hence:

$$
A_{w_{1}, w_{2}}^{\tau_{1}, \tau_{2}}(r, B) \leq C|B|^{\frac{1}{n}+\frac{1}{\tau_{1}}-\frac{1}{\tau_{2}}} d_{B} d_{B}^{\frac{\tau_{2}\left(1-\tau_{2}^{\prime}\right)}{\tau_{2}^{\prime}}}=C|B|^{\frac{\gamma_{2}-\gamma_{1}}{n}} \leq C,
$$

where we used condition $(2.4)$ and $\gamma_{2}-\gamma_{1} \geq 0$.

On the other hand, if $r_{B} \geq d_{B}$, we may assume that $B$ is centered at the origin, and proceed integrating in spherical coordinates:

$$
\begin{aligned}
A_{w_{1}, w_{2}}^{\tau_{1}, \tau_{2}}(r, B) & \leq C|B|^{\frac{1}{n}+\frac{1}{\tau_{1}}-\frac{1}{\tau_{2}}}\left(\frac{1}{|B|} \int_{0}^{r_{B}} \rho^{\gamma_{1} r \tau_{1}+n-1} \mathrm{~d} \rho\right)^{\frac{1}{r \tau_{1}}}\left(\frac{1}{|B|} \int_{0}^{r_{B}} \rho^{\gamma_{2} r \tau_{2}\left(1-\tau_{2}^{\prime}\right)+n-1} \mathrm{~d} \rho\right)^{\frac{1}{r \tau_{2}^{\prime}}} \\
& \leq C|B|^{\frac{1}{n}+\frac{1}{\tau_{1}}-\frac{1}{\tau_{2}}-\frac{1}{r \tau_{1}}-\frac{1}{r \tau_{2}^{\prime}}} r_{B}^{\gamma_{1}+\frac{n}{r \tau_{1}}+\gamma_{2} \frac{\tau_{2}\left(1-\tau_{2}^{\prime}\right)}{\tau_{2}^{\prime}}+\frac{n}{r \tau_{2}^{\prime}}} \\
& =C r_{B}^{1+\frac{n}{\tau_{1}}-\frac{n}{\tau_{2}}-\frac{n}{r \tau_{1}}-\frac{n}{r \tau_{2}^{\prime}}+\gamma_{1}+\frac{n}{r \tau_{1}}-\gamma_{2}+\frac{n}{r \tau_{2}^{\prime}}}=C r_{B}^{1+\frac{n}{\tau_{1}}-\frac{n}{\tau_{2}}+\gamma_{1}-\gamma_{2}}=C .
\end{aligned}
$$

In the last step, we used (2.4). The other conditions on the statement give the integrability requierements for this estimate, provided that $r>1$ is small enough.

Taking $\left|x-x_{0}\right|$ instead of $|x|, \gamma_{1}=\alpha-1, \gamma_{2}=\alpha$ and $\tau_{1}=\tau_{2}=2$ in Theorem 2.2 we obtain the imbedding $H_{0, \alpha}^{1}(\Omega) \subset L_{\alpha-1}^{2}$, for every $\alpha>1-\frac{n}{2}$. This induces us to work with Kondratiev type spaces, rather than with standard Sobolev ones. We define the Kondratiev space $K_{\eta}^{\ell}(\Omega)$, formed by functions $v$ such that:

$$
\|v\|_{K_{\eta}^{\ell}(\Omega)}^{2}=\sum_{|\gamma| \leq \ell} \int_{\Omega} d(x)^{2(\eta+|\gamma|)}\left|D^{\gamma} v(x)\right|^{2} \mathrm{~d} x<\infty .
$$

Our error estimates assume that the solution $u$ of (2.3) belongs to $K_{\eta}^{2}$ for certain values of $\eta$. This is indeed the case for the most interesting examples:

Example 2.3. Consider $f=\delta_{x_{0}}$. It is proven in Theorem 4.7 of [1], that $\delta_{x_{0}} \in\left(H_{0,-\alpha}^{1}(\Omega)\right)^{\prime}$ for

$$
\frac{n}{2}-1<\alpha<\frac{n}{2}
$$

Recall that the fundamental solution for problem (1.1) is given by:

$$
\bar{u}(x)= \begin{cases}-\frac{1}{2 \pi} \log \left(\left|x-x_{0}\right|\right) & \text { if } n=2 \\ \frac{1}{4 \pi\left|x-x_{0}\right|} & \text { if } n=3 .\end{cases}
$$

Hence, it is easy to check that the solution $u$ of problem (2.3) with $f=\delta_{x_{0}}$ belongs to $K_{\eta}^{2}$ for every $\eta>\frac{n}{2}-2$. 
Similarly:

Example 2.4. Consider $f(x)=\left|x-x_{0}\right|^{-s}$ for $s<n$. For $v \in H_{0,-\alpha}^{1}(\Omega)$ apply Theorem 2.2 with $\tau_{1}=1$, $\tau_{2}=2, \gamma_{1}=-s, \gamma_{2}=\beta$, and $\beta=s-\frac{n}{2}-1$, obtaining:

$$
\left|\int_{\Omega} f(x) v(x) \mathrm{d} x\right| \leq C\left\|\left|x-x_{0}\right|^{-\beta} \nabla v\right\|_{L^{2}(\Omega)} \leq C\|v\|_{H_{-\alpha}^{1}(\Omega)}
$$

provided that $\alpha \geq \beta$. Hence, for $s-\frac{n}{2}-1 \leq \alpha<\frac{n}{2}, f \in\left(H_{0,-\alpha}^{1}(\Omega)\right)^{\prime}$, and Theorem 2.1 can be applied. Observe that for $s \leq \frac{n}{2}+1, \alpha=0$ can be taken, so $f \in H^{-1}(\Omega)$. In this case, our results on weighted spaces still hold, but they are less interesting, since a standard analysis in $H_{0}^{1}$ is available. On the other hand for $\frac{n}{2}+1<s<n$ $(n=3)$, problem (1.1) is singular and admits our weighted formulation and estimates. Moreover, in this case $|u(x)| \sim|x|^{2-s}$ and $u \in K_{\eta}^{2}(\Omega)$ for $\eta>s-\frac{7}{2}$.

\section{Weighted a priori ESTIMATES}

For proving the stability of the Galerkin projection in $H_{\alpha}^{1}$, we will also need weighted a priori estimates for problem (1.1) with $f \in L_{\omega}^{p}(\Omega)$ for $\omega \in A_{p}(\Omega)$. In particular, we prove:

Theorem 3.1. Let $\Omega$ be a $\mathcal{C}^{1,1}$ domain or a convex polygon o polyhedron. Then there is an interval I around $p=2$ such that for $p \in I$ and every $\omega \in A_{p}(\Omega)$, if $f \in L_{\omega}^{p}(\Omega)$, problem (1.1) admits a unique weak solution $u \in W_{0, \omega}^{2, p}(\Omega)$ that satisfies:

$$
\|u\|_{W_{\omega}^{2, p}(\Omega)} \leq C\|f\|_{L_{\omega}^{p}(\Omega)} .
$$

The ideas for proving this result were suggested by Prof. R. Duran, and follows the same arguments used in [25] for obtaining Theorem 2.1. We only give a brief sketch of the proof. The main idea is to take advantage of a general weighted estimate for smooth domains in the interior of $\Omega$, and of an unweighted estimate (with less smoothness requirements), near the boundary. In particular we have:

Theorem 3.2. Given $\Omega \in \mathbb{R}^{n}$, such that $\partial \Omega$ is of class $\mathcal{C}^{\ell}$ with $\ell=6$ for $n=3$ and $\ell=9$ for $n=2,1<p<\infty$, $\omega \in A_{p}$ and $f \in L_{\omega}^{p}(\Omega)$ there is a unique solution of $-\Delta u=f, u=0$ on $\partial \Omega$, that satisfies:

$$
\|u\|_{W_{\omega}^{2, p}(\Omega)} \leq C\|f\|_{L_{\omega}^{p}(\Omega)} .
$$

Proof. See [9].

Theorem 3.3. Let $\Omega$ a $\mathcal{C}^{1,1}$ domain of a convex polygon or polyhedron. There is an interval $I$ around $p=2$ such that for $p \in I$ if $f \in L^{p}(\Omega)$ problem (1.1) admits a unique solution $u \in W^{2, p}(\Omega)$ satisfying:

$$
\|u\|_{W^{2, p}(\Omega)} \leq C\|f\|_{L^{p}(\Omega)} .
$$

Proof. For $\mathcal{C}^{1,1}$ domains, the result holds for $I=(1, \infty)$ (see [15], Thm. 9.15, Lem. 9.17). For convex polygons and polyhedra, singularities arises on the vertices of the domain that prevent the result to hold for large values of $p$. However, for convex polygons it holds for $I=\left(1, p_{0}\right)$ with $p_{0}$ depending on the domain. In particular, $p_{0} \rightarrow \infty$ if the largest interior angle tends to $\frac{\pi}{2}$, and $p_{0} \searrow 2$ if the largest interior angle tends to $\pi$ (see [16], Thm. 4.3.2.4, Rem. 4.3.2.5). On the other hand, for convex polyhedrons, $I=\left(\frac{6}{5}, p_{0}\right)$ with $p_{0}>2$ depending on the domain (see [23]).

Now, we can prove the desired result: 
Proof of Theorem 3.1. Following [25], we begin proving:

$$
\|u\|_{W_{\omega}^{2, p}(\Omega)} \leq C\left\{\|f\|_{L_{\omega}^{p}(\Omega)}+\|u\|_{W^{1, p}(G)}\right\} .
$$

Take two cut-off functions $\psi_{i}+\psi_{\partial}=1$ such that $\psi_{i} \equiv 1$ in a neighbourhood of $\Omega \backslash G$ and $\operatorname{supp}\left(\nabla \psi_{\mathrm{i}}\right) \cup$ $\operatorname{supp}\left(\nabla \psi_{\partial}\right) \subset \overline{\mathrm{G}}$. Moreover, we assume that $\Omega_{i}=\operatorname{supp}\left(\psi_{\mathrm{i}}\right)^{\circ}$ satisfies the smoothness condition on Theorem 3.2. Define $u_{i}=u \psi_{i}$ and $u_{\partial}=u \psi_{\partial}$. In this way, we have that $u_{i}$ is supported on a smooth subdomain of the interior or $\Omega$, whereas $u_{\partial}$ is supported on $G$, near $\partial \Omega$. Now, for every $\varphi \in C_{0}^{\infty}\left(\Omega_{i}\right)$ :

$$
\begin{aligned}
\int_{\Omega_{i}} \nabla u_{i} \nabla \varphi & =\int_{\Omega_{i}} \nabla u \nabla\left(\psi_{i} \varphi\right)-\int_{\Omega_{i}} \varphi \nabla u \cdot \nabla \psi_{i}+\int_{\Omega_{i}} u \nabla \psi_{i} \cdot \nabla \varphi \\
& =\int_{\Omega_{i}}\left(f \psi_{i}-\nabla u \cdot \nabla \psi_{i}-\operatorname{div}\left(\mathrm{u} \nabla \psi_{\mathrm{i}}\right)\right) \varphi
\end{aligned}
$$

Hence, $u_{i}$ is a weak solution of (1.1) on $\Omega_{i}$ and right hand side $g=f \psi_{i}-\nabla u \cdot \nabla \psi_{i}-\operatorname{div}\left(u \nabla \psi_{\mathrm{i}}\right)$. Consequently, Theorem 3.2 gives:

$$
\begin{aligned}
\left\|u_{i}\right\|_{W_{\omega}^{2, p}\left(\Omega_{i}\right)} & \leq C\|g\|_{L_{\omega}^{p}\left(\Omega_{i}\right)} \leq C\left\{\|f\|_{L_{\omega}^{p}(\Omega)}+\left\|\nabla u \nabla \psi_{i}+\operatorname{div}\left(\mathrm{u} \nabla \psi_{\mathrm{i}}\right)\right\|_{L_{\omega}^{\mathrm{p}}\left(\Omega_{\mathrm{i}}\right)}\right\} \\
& \leq C\left\{\|f\|_{L_{\omega}^{p}(\Omega)}+\|u\|_{W_{\omega}^{1, p}(G)}\right\} \leq C\left\{\|f\|_{L_{\omega}^{p}(\Omega)}+\|u\|_{W^{1, p}(G)}\right\}
\end{aligned}
$$

where we used that $\operatorname{supp}\left(\nabla \psi_{i}\right) \subset \bar{G}$ and that the weight $\omega$ can go out of the norm restricted to $G$.

For $u_{\partial}$ a similar approach can be applied. However it is convenient to integrate on $\Omega$ instead of $G$ (or $\left.\operatorname{supp}\left(\psi_{\partial}\right)\right)$. We obtain:

$$
\int_{\Omega} \nabla u_{\partial} \nabla \varphi=\int_{\Omega}\left(f \psi_{\partial}-\nabla u \cdot \nabla \psi_{\partial}-\operatorname{div}\left(\mathrm{u} \nabla \psi_{\partial}\right)\right) \varphi
$$

Both $u_{\partial}$ and $g_{\partial}=f \psi_{\partial}-\nabla u \cdot \nabla \psi_{\partial}-\operatorname{div}\left(\mathrm{u} \nabla \psi_{\partial}\right)$ are supported on $G \subset \Omega$, and $u_{\partial}$ can be regarded as the solution of problem (1.1) with right hand side $g_{\partial}$, on $\Omega$. Since $\Omega$ satisfies the regularity conditions on Theorem 3.3, we apply the estimate, and recall that $\operatorname{supp}\left(\psi_{\partial}\right) \subset \mathrm{G}$, obtaining:

$$
\left\|u_{\partial}\right\|_{W^{2, p}(\Omega)} \leq C\left\|g_{\partial}\right\|_{L^{p}(\Omega)}=C\left\|g_{\partial}\right\|_{L^{p}(G)} \leq C\left\{\|f\|_{L^{p}(G)}+\|g\|_{W^{1, p}(G)}\right\} \leq C\left\{\|f\|_{L_{\omega}^{p}(G)}+\|g\|_{W^{1, p}(G)}\right\} .
$$

The proof of (3.2) is completed by observing that:

$$
\|u\|_{W_{\omega}^{2, p}(\Omega)} \leq\left\|u_{i}\right\|_{W_{\omega}^{2, p}\left(\Omega_{i}\right)}+\left\|u_{\partial}\right\|_{W_{\omega}^{2, p}(G)} \leq\left\|u_{i}\right\|_{W_{\omega}^{2, p}(\Omega)}+C\left\|u_{\partial}\right\|_{W^{2, p}(G)} .
$$

Now, reasoning by contradiction and assuming $p \in I, p \geq 2$, if the desired estimate does not hold, there are sequences $u_{k}, f_{k}$ such that $u_{k}$ is a weak solution of problem (1.1) with right hand side $f_{k}$, and $\left\|u_{k}\right\|_{W_{\omega}^{2, p}(\Omega)}=1$ while $f_{k} \rightarrow 0$ in $L_{\omega}^{p}(\Omega)$. We can take a sub-sequence (relabeled $u_{k}$ ) with $u_{k} \rightarrow u \in W_{0, \omega}^{2, p}(\Omega)$. The limit $u$ is a weak solution of (1.1) with right hand side $f=0$. We apply Lemma 6 of [25] where it is shown that for $p \geq 2$ the unique solution of this problem is $u=0$. Taking into account the compact embedding of $W_{0, \omega}^{2, p}(\Omega)$ into $W_{0, \omega(\Omega)}^{1, p}$, we have that $u_{k} \rightarrow 0$ in $W_{\omega}^{1, p}(\Omega)$. Hence we have:

$$
1 \leq\|u\|_{W_{\omega}^{2, p}(\Omega)} \leq\|u\|_{W^{1, p}(G)}=0
$$

which is absurd, proving the estimate.

The existence of solution can be deduced from the estimate by an approximation argument. Finally, a duality argument completes the proof for $p \in I, p<2$. The reader can see the details in [25].

Remark 3.4. The restrictions that we need to impose on our domains are due to Theorem 3.1, which inherits them from Theorem 3.3. Some other domains could be considered, as long as they satisfy Theorem 3.3 for some values of $p$ near $p=2$. See the Proof of Claim 1 below, where Theorem 3.1 is used. 


\section{Stability of the Galerkin Projection}

For the discrete problem, we consider $\mathcal{T}_{h}$ a triangulation of $\Omega$ formed by shape-regular elements $T$, graded towards the singular point $x_{0}$. We choose $h=2^{-m}$ for some $m \in \mathbb{N}$ and denote $r_{T}=\operatorname{dist}\left(T, x_{0}\right), h_{T}=\operatorname{diam}(T)$. Fixing a value $\mu_{0}>0$, we take $\mu_{0}<\mu \leq 1$, and build a mesh such that:

$$
h_{T} \sim \begin{cases}h^{\frac{1}{\mu}} & \text { if } r_{T}=0 \\ h r_{T}^{1-\mu} & \text { if } 0<r_{T} \leq 1 \\ h & \text { if } r_{T}>1 .\end{cases}
$$

Remark 4.1. The number of elements of a mesh graded according to (4.1) towards a point is $N \leq C_{\mu} h^{-n}$ (see [3], Rem. 3.1), as is the case for quasi-uniform meshes with elements of diameter $\sim h$. The constant $C_{\mu}$ tends to infinity as $\mu \rightarrow 0$, hence the restriction $\mu>\mu_{0}$ in order to preserve the relationship between the parameter $h$ and the number of degrees of freedom of the numerical problem.

$\mathcal{P}_{k}$ stands for the set of polynomials of degree at most $k$. We define the discrete space:

$$
V_{h}^{k}=\left\{v \in H_{0}^{1}(\Omega):\left.v\right|_{T} \in \mathcal{P}_{k}, \forall T \in \mathcal{T}_{h}\right\}
$$

For simplicity, we focus on linear polynomials, so we drop the index $k$ and write $V_{h}$.

We denote $u \in H_{0, \alpha}^{1}$ the unique solution of problem (2.3) and $u_{h}$ its Galerkin projection on $V_{h}$ :

$$
\left(\nabla u_{h}, \nabla v_{h}\right)=\left(\nabla u, \nabla v_{h}\right) \quad \forall v_{h} \in V_{h} .
$$

The goal of this section is to prove that:

Theorem 4.2. Let $\Omega$ be a $\mathcal{C}^{1,1}$ domain, or a convex polygon or polyhedron, $-\frac{n}{2}<\alpha<\frac{n}{2}, u \in H_{0, \alpha}^{1}(\Omega)$ and $u_{h}$ its Galerkin projection on a mesh graded according to (4.1). Then:

$$
\left\|\nabla u_{h}\right\|_{L_{\alpha}^{2}(\Omega)} \leq C\|\nabla u\|_{L_{\alpha}^{2}(\Omega)} .
$$

Theorem 4.2 in turn implies the best approximation property:

$$
\left\|\nabla\left(u-u_{h}\right)\right\|_{L_{\alpha}^{2}(\Omega)} \leq C \inf _{v_{h} \in V_{h}}\left\|\nabla\left(u-v_{h}\right)\right\|_{L_{\alpha}^{2}(\Omega)},
$$

which is the key for obtaining a priori error estimates.

We derive Theorem 4.2 as an application of a more general stability result given in Theorem 4.3 below (see also Cor. 4.4). Our proof relies on two main assumptions on the mesh. We state these assumptions as claims and leave the proofs for later, since they requiere some technical efforts.

The main ideas for setting the stage are based on the classical estimates in $W^{1, \infty}$ ([7], Chap. 8). Let us denote for every $z \in \Omega, T_{z} \in \mathcal{T}_{h}$ an element such that $z \in T_{z}, h_{z}=\operatorname{diam}\left(T_{z}\right)$ and $\eta_{z} \in C_{0}^{\infty}\left(T_{z}\right)$ a smooth approximation of a Dirac delta, such that:

$$
\int \eta_{z} P \mathrm{~d} x=P(z) \quad \forall P \in \mathcal{P}_{k}, \quad\left|D^{\gamma} \eta_{z}\right| \leq h_{z}^{-n-|\gamma|} .
$$

We also define the smoothen weight:

$$
\sigma_{z}(x)=\left(|x-z|^{2}+\left(t h_{z}\right)^{2}\right)^{\frac{1}{2}},
$$

where $t \geq 1$ is a parameter to be determined later. It is easy to check that:

$$
\left|D^{\gamma} \sigma_{z}^{\lambda}(x)\right| \leq C \sigma_{z}^{\lambda-|\gamma|}(x)
$$


for every $\lambda \in \mathbb{R}$.

Let $\partial$ stand for a directional derivative, and $g$ for the solution of the problem: $g \in H_{0}^{1}(\Omega)$, $(\nabla g, \nabla \varphi)=\left(-\partial \eta_{z}, \varphi\right)$, for every $\varphi \in H_{0}^{1}(\Omega) . g_{h}$ is the Galerkin projection of $g$.

Thence, using repeteadly the Galerkin orthogonality we have:

$$
\begin{aligned}
\partial u_{h}(z) & =\left(\eta_{z}, \partial u_{h}\right)=\left(-\partial \eta_{z}, u_{h}\right)=\left(\nabla g, \nabla u_{h}\right) \\
& =(\nabla g, \nabla u)-\left(\nabla g, \nabla\left(u-u_{h}\right)\right) \\
& =\left(-\partial \eta_{z}, u\right)-\left(\nabla\left(g-g_{h}\right), \nabla u\right) \\
& =\left(\eta_{z}, \partial u\right)-\left(\nabla\left(g-g_{h}\right), \nabla u\right) .
\end{aligned}
$$

Taking $\lambda \in \mathbb{R}$ and applying Hölder's inequality, we obtain:

$$
\left|\nabla u_{h}(z)\right| \leq \frac{1}{h_{z}^{n}} \int_{T_{z}}|\nabla u(x)| \mathrm{d} x+\left\|h_{z}^{-\frac{\lambda}{2}} \sigma_{z}^{\frac{n+\lambda}{2}} \nabla\left(g-g_{h}\right)\right\|_{L^{2}(\Omega)}\left\|h_{z}^{\frac{\lambda}{2}} \sigma_{z}^{-\frac{n+\lambda}{2}} \nabla u\right\|_{L^{2}(\Omega)} .
$$

The rest of the argument follows as in [12]. We recall the details for the sake of completeness. We begin observing that the first term on the right hand side of (4.6) is bounded by $\mathcal{M} \nabla u(x)$. For the second term, we assume that:

Claim 1. The domain $\Omega$ and its triangulation $\mathcal{T}_{h}$ are such that there exists $t \geq 1$ large enough and $\lambda>0$ such that:

$$
\left\|h_{z}^{-\frac{\lambda}{2}} \sigma_{z}^{\frac{n+\lambda}{2}} \nabla\left(g-g_{h}\right)\right\|_{L^{2}(\Omega)} \leq C
$$

for a constant $C$ independent of $z$.

Now, we can square (4.6), multiply by a weight $\omega$ and integrate, obtaining:

$$
\left\|\nabla u_{h}\right\|_{L_{\omega}^{2}(\Omega)}^{2} \leq C\left\{\|\mathcal{M} \nabla u\|_{L_{\omega}^{2}(\Omega)}^{2}+h_{z}^{\lambda} \int_{\Omega}\left\|\sigma_{z}^{-\frac{n+\lambda}{2}} \nabla u\right\|_{L^{2}(\Omega)}^{2} \omega(z) \mathrm{d} z\right\} .
$$

The second needed result is, then:

Claim 2. The domain $\Omega$ and its triangulation $\mathcal{T}_{h}$ are such that there exist $t \geq 1$ large enough and $\lambda>0$ such that:

$$
\int_{\Omega}\left\|h_{z}^{\frac{\lambda}{2}} \sigma_{z}^{-\frac{n+\lambda}{2}} \nabla u\right\|_{L^{2}(\Omega)}^{2} \omega(z) \mathrm{d} z \leq C\|\nabla u\|_{L_{\mathcal{M} \omega}^{2}(\Omega)}^{2}
$$

for a constant $C$ independent of $z$.

In this way, we conclude:

$$
\left\|\nabla u_{h}\right\|_{L_{\omega}^{2}(\Omega)}^{2} \leq C\left\{\|\mathcal{M} \nabla u\|_{L_{\omega}^{2}(\Omega)}^{2}+\|\nabla u\|_{L_{\mathcal{M} \omega}^{2}(\Omega)}^{2}\right\} .
$$

Now if we take $\omega \in A_{1}$, we have $\mathcal{M} \omega \leq[\omega]_{A_{1}} \omega$, which gives an estimate for the second term, whereas thanks to the continuity of the maximal operator in $L_{\omega}^{2}$, with weights in $A_{2} \supset A_{1}$, the first term can be also be bounded, obtaining:

$$
\left\|\nabla u_{h}\right\|_{L_{\omega}^{2}(\Omega)}^{2} \leq C\|\nabla u\|_{L_{\omega}^{2}(\Omega)} .
$$

This results can be extended to weights $\omega$ such that $\omega^{-1} \in A_{1}$ (see [12], Cor. 3.3), proving:

Theorem 4.3. Assume $\Omega$ and $\mathcal{T}_{h}$ are such that Claims 1 and 2 hold, $\omega \in A_{1}$ or $\omega^{-1} \in A_{1}$. Then:

$$
\left\|\nabla u_{h}\right\|_{L_{\omega}^{2}(\Omega)} \leq C\|\nabla u\|_{L_{\omega}^{2}(\Omega)} .
$$


It is interesting to notice that, as in [12], this result can be extended for weights in $A_{1}$ and $2<p<\infty$ :

Corollory 4.4. If $\omega \in A_{1}$ then for $2<p<\infty$ there exists a constant $C$ depending only on $[\omega]_{A_{1}}$ such that:

$$
\left\|\nabla u_{h}\right\|_{L_{\omega}^{p} \Omega} \leq C\|\nabla u\|_{L_{\omega}^{p}(\Omega)} .
$$

Proof. The result follows by extrapolation. Theorem 4.3 says that $\left\|\nabla u_{h}\right\|_{L_{\omega}^{2}(\Omega)} \leq\|\nabla u\|_{L_{\omega}^{2}(\Omega)}$ for every $\omega \in A_{1}$. Applying Corollary 3.5 of [13] (taking $p_{0}=2, s_{0}=1$ ), we have that:

$$
\left\|\nabla u_{h}\right\|_{L_{\omega}^{p}(\Omega)} \leq C\|\nabla u\|_{L_{\omega}^{p}(\Omega)},
$$

for $2<p<\infty$, and every weight $\omega \in A_{\frac{p}{2}}$. Since $A_{1} \subset A_{\frac{p}{2}}$ and $[\omega]_{A_{\frac{p}{2}}} \leq[\omega]_{A_{1}}$ for every $p>2$, the result follows.

In [12], Claims 1 and 2 are proven for smooth domains and convex polygons and polyhedrons and for quasiuniform meshes. Here we are interested in graded meshes, and in weights of the form $d(x)^{2 \alpha}$. Observe that, (2.2) implies that if $-\frac{n}{2}<\alpha<\frac{n}{2}, d(x)^{2 \alpha} \in A_{2}$ and either $d(x)^{2 \alpha} \in A_{1}$ or $d(x)^{-2 \alpha} \in A_{1}$. Hence, Theorem 4.2 is a corollary of Theorem 4.3. For completing its proof, we need to verify that Claims 1 and 2 hold on our graded meshes.

The key of the proof given in [12], following [17], is to consider the auxiliary weight $\sigma_{z}$ over a partition of the domain in concentric rings centered at $z$, where $\sigma_{z}$ can be regarded as constant. Our arguments follow closely [21]: we need two partitions of the domain. The first one, agrees with the graduation of the mesh, and is, therefore centered at $x_{0}$. The second one is essentially the one used in [12].

For the first partition, recalling that $h=2^{-m}$, we define

$$
\kappa=2^{-\frac{1}{\mu}},
$$

and take:

$$
\begin{aligned}
& L_{0}=\left\{x \in \Omega: 0 \leq d(x) \leq \kappa^{m}\right\} \\
& L_{j}=\left\{x \in \Omega: \kappa^{m-j+1}<d(x) \leq \kappa^{m-j}\right\} \quad j=1, \ldots, J
\end{aligned}
$$

where $J$ is chosen so that $\Omega=\cup_{j=0}^{J} L_{j}$ and $L_{J} \neq \emptyset$. We denote $h_{j}$ the diameter of the triangles or tetrahedra in $L_{j}$. The following properties are easy to check:

$$
\begin{aligned}
h & =\kappa^{m \mu} \\
h_{j} & \sim \kappa^{m-j(1-\mu)} .
\end{aligned}
$$

The radius of the ring $L_{j}$ is $\sim \kappa^{m-j}$.

It is also easy to check that:

$$
\max _{x \in T} \sigma_{z}^{\lambda}(x) \leq C \min _{x \in T} \sigma_{z}^{\lambda}(x) \quad \forall T \in \mathcal{T}_{h} .
$$

This allows us to define $\sigma_{z, T}$ an average value of $\sigma_{z}$ for each $T \in \mathcal{T}_{h}$ such that $\sigma_{z}(x) \sim \sigma_{z, T}$ for every $x \in T$. Moreover, we have:

$$
h_{T} \leq C \sigma_{z, T}
$$

For verifying this, let us assume that $z \in L_{j}$, and $T \subset L_{k} \neq \emptyset$. Then, if $k \leq j+1$ we have that $h_{T}=h_{k} \leq$ $C h_{j}=C h_{z} \leq C \sigma_{z, T}$. On the other hand, if $k>j+1$, the distance between $x$ and $z$ is greater than the width of the ring $L_{j+1}$, so: $|x-z| \geq h_{j+1}$, and thence $h_{T} \sim h_{k} \leq C \sigma_{z, T}$. 
In order to control the auxiliary weight $\sigma_{z}$, we need to combine the partition $\left\{L_{j}\right\}_{j}$ with a second one: given a point $x \in \Omega$, let us define the following sets, centered at $x$.

$$
\begin{aligned}
& B_{x}=\left\{y \in \Omega:|y-x|<h_{x}\right\} \\
& \Omega_{k}^{x}=\left\{y \in \Omega: 2^{k-1} h_{x}<|y-x| \leq 2^{k} h_{x}\right\}, \quad k=1, \ldots, K
\end{aligned}
$$

These sets will allow us to estimate $h_{z}$ and $|x-z|$ (and hence $\sigma_{z}$ ), for every $z$. The decomposition used in [12] is a little simpler, since only quasi-uniform meshes are considered, where $h_{x} \sim h$ for every $x$.

Before tackling Claims 1 and 2 we prove an auxiliary lemma that connects our two partitions:

Lemma 4.5. Let $\bar{y} \in L_{\ell}$ and $j>\ell+1$. If $\Omega_{k}^{\bar{y}} \cap L_{j} \neq \emptyset$, then:

$$
\ell+\frac{1}{\mu}(j-\ell-1) \leq k \leq 2+\ell+\frac{1}{\mu}(j-\ell)
$$

Proof. Consider $\xi \in L_{j}$ for $j>\ell+1$. Then:

$$
\begin{aligned}
& |\xi-\bar{y}| \leq\left|\xi-x_{0}\right|+\left|\bar{y}-x_{0}\right| \leq \kappa^{m-j}+\kappa^{m-\ell} \leq 2 \kappa^{m-j} \\
& |\xi-\bar{y}| \geq\left|\xi-x_{0}\right|-\left|\bar{y}-x_{0}\right| \geq \kappa^{m-j+1}-\kappa^{m-\ell} \geq \kappa^{m-j+1}(1-\kappa) .
\end{aligned}
$$

Hence,

$$
L_{j} \subset G_{j}:=\left\{y: \kappa^{m-j+1}(1-\kappa)<|y-\bar{y}|<2 \kappa^{m-j}\right\} .
$$

We now consider the set $G_{j}$, since it is centered at $\bar{y}$. If $\Omega_{k}^{\bar{y}} \cap L_{j} \neq \emptyset$, then $\Omega_{k}^{\bar{y}} \cap G_{j} \neq \emptyset$, so:

$$
\kappa^{m-j+1}(1-\kappa) \leq 2^{k} h_{\bar{y}} \quad \text { and } \quad 2^{k-1} h_{\bar{y}} \leq 2 \kappa^{m-j},
$$

and these conditions lead to the result.

We begin by proving Claim 2, which is easier.

Proof of Claim 2 for graded meshes. By Fubini's lemma we have:

$$
\int_{\Omega} \int_{\Omega} h_{z}^{\lambda} \sigma_{z}^{-n-\lambda}(x)|\nabla u(x)|^{2} \mathrm{~d} x \omega(z) \mathrm{d} z=\int_{\Omega}|\nabla u(x)|^{2} \int_{\Omega} h_{z}^{\lambda} \sigma_{z}^{-n-\lambda}(x) \omega(z) \mathrm{d} z \mathrm{~d} x .
$$

Hence, we only need to prove:

$$
\int_{\Omega} h_{z}^{\lambda} \sigma_{z}^{-n-\lambda}(x) \omega(z) \mathrm{d} z \leq C \mathcal{M} w(x) .
$$

We study this integral along the decomposition of $\Omega$ induced by the sets $B_{x}$ and $\Omega_{k}^{x}$. We take $\ell$ such that $x \in L_{\ell}$.

If $z \in B_{x}, h_{z} \sim h_{x}$. Hence:

$$
\int_{B_{x}} h_{z}^{\lambda} \sigma_{z}^{-n-\lambda}(x) \omega(z) \mathrm{d} z \leq C t^{-\lambda-n} \frac{1}{h_{x}^{n}} \int_{B_{x}} \omega(z) \mathrm{d} z \leq C \mathcal{M} \omega(x) .
$$


On the other hand, for $z \in B_{x}^{c}$, we consider two separate cases: $z \in \cup_{j \leq \ell+1} L_{j}$ and $z \in \cup_{j>\ell+1} L_{j}$.

$$
\begin{aligned}
\int_{B_{x}^{c} \cap\left(\cup_{j \leq \ell+1} L_{j}\right)} h_{z}^{\lambda} \sigma_{z}^{-n-\lambda}(x) \omega(z) \mathrm{d} z \leq \sum_{j \leq \ell+1} h_{j}^{\lambda} \int_{B_{x}^{c} \cap L_{j}} \frac{\omega(z)}{|x-z|^{n+\lambda}} \mathrm{d} z \\
=\sum_{j \leq \ell+1} h_{j}^{\lambda} \sum_{k} \int_{B_{x}^{c} \cap L_{j} \cap \Omega_{k}^{x}} \frac{\omega(z)}{|x-z|^{n+\lambda}} \mathrm{d} z \\
\leq C \sum_{j \leq \ell+1} h_{j}^{\lambda} \sum_{k} \frac{1}{\left(2^{k} h_{x}\right)^{n+\lambda}} \int_{\Omega_{k}^{x}} \omega(z) \mathrm{d} z \\
\leq C \sum_{j \leq \ell+1} h_{j}^{\lambda} \sum_{k} \frac{1}{\left(2^{k} h_{x}\right)^{\lambda}} \mathcal{M} \omega(x) \leq C \sum_{j \leq \ell+1} \frac{h_{j}^{\lambda}}{h_{x}^{\lambda}} \mathcal{M} \omega(x) \\
\leq C \mathcal{M} \omega(x)
\end{aligned}
$$

where, in the last step, we used that $\sum_{j \leq \ell+1} h_{j} \leq h_{\ell} \sim h_{x}$.

For the rest of the estimate, we use Lemma 4.5, with $\bar{y}=x$ :

$$
\begin{aligned}
\int_{B_{x}^{c} \cap\left(\bigcup_{j>\ell+1} L_{j}\right)} h_{z}^{\lambda} \sigma_{z}^{-n-\lambda}(x) \omega(z) \mathrm{d} z \leq \sum_{j>\ell+1} h_{j}^{\lambda} \int_{B_{x}^{c} \cap L_{j}} \frac{\omega(z)}{|x-z|^{n+\lambda}} \mathrm{d} z \\
\quad=\sum_{j>\ell+1} h_{j}^{\lambda} \sum_{k=\ell+\frac{1}{\mu}(j-\ell-1)}^{2+\ell+\frac{1}{\mu}(j-\ell)} \int_{B_{x}^{c} \cap L_{j} \cap \Omega_{k}^{x}} \frac{\omega(z)}{|x-z|^{n+\lambda}} \mathrm{d} z \\
\leq C \sum_{j>\ell+1} h_{j}^{\lambda} \sum_{k=\ell+\frac{1}{\mu}(j-\ell-1)}^{2+\ell+\frac{1}{\mu}(j-\ell)} \frac{1}{\left(2^{k} h_{x}\right)^{n+\lambda}} \int_{\Omega_{k}^{x}} \omega(z) \mathrm{d} z \\
\leq C \sum_{j>\ell+1} h_{j}^{\lambda} h_{x}^{-\lambda} \sum_{k=\ell+\frac{1}{\mu}(j-\ell-1)}^{2+\ell+\frac{1}{\mu}(j-\ell)} 2^{-\lambda k} \mathcal{M} \omega(x) \\
\leq C h_{\ell}^{-\lambda} \sum_{j>\ell+1} \kappa^{\lambda(m-(1-\mu) j)} \kappa^{\lambda(\mu \ell+j-\ell-1+\mu)} \mathcal{M} \omega(x) \\
\leq C h_{\ell}^{-\lambda} h_{\ell}^{\lambda} \kappa^{\lambda(\mu-1)} \sum_{j>\ell+1} \kappa^{\mu j} \mathcal{M} \omega(x) \leq C \mathcal{M} \omega(x) .
\end{aligned}
$$

This completes the proof of the result. Observe that the constant $C$ may depend on $\kappa^{-1}$, so we used the condition $\mu>\mu_{0}$.

The proof of Claim 1 is rather cumbersome. Several technical lemmas are needed.

The first step in our way to proving Claim 1 is Lemma 4.6. For $v \in H^{2}(\Omega)$, we denote $v_{I} \in V_{h}$ such that $\left.v_{I}\right|_{T}$ is the Lagrange interpolator of $v$ in the element $T \in \mathcal{T}_{h}$.

Lemma 4.6. Let $g_{I} \in V_{h}$ the Lagrange interpolation of $g$. There is a constant $C$ such that:

$$
\int_{\Omega} \sigma_{z}^{n+\lambda}\left|\nabla\left(g-g_{h}\right)\right|^{2} \leq C\left\{\int_{\Omega} \sigma_{z}^{n+\lambda-2}\left(g-g_{h}\right)^{2}+\int_{\Omega} \sigma_{z}^{n+\lambda-2}\left(g-g_{I}\right)^{2}+\int_{\Omega} \sigma_{z}^{n+\lambda}\left|\nabla\left(g-g_{I}\right)\right|^{2}\right\} .
$$

Proof. This result is proven in [7] and in [21]. In the statement of Proposition 8.3.1 from [7] the hypothesis of $W^{1, p}$ regularity for some $p>n$ is assumed, but not used, whereas in Lemma 5.1 of [21] only the twodimensional case in considered. Our proof follows [21], since the dimension is irrelevant to the argument. Let us 
denote $e=g-g_{h}, \psi=\sigma_{z}^{n+\lambda}\left(g_{I}-g_{h}\right)$ and $\psi_{I}$ the Lagrange interpolant of $\psi$. Then, we have:

$$
\begin{aligned}
\int_{\Omega} \sigma_{z}^{n+\lambda}|\nabla e|^{2} & =\int_{\Omega} \sigma_{z}^{n+\lambda} \nabla\left(g-g_{h}\right) \cdot \nabla\left(g-g_{I}\right)+\int_{\Omega} \sigma_{z}^{n+\lambda} \nabla\left(g-g_{h}\right) \cdot \nabla\left(g_{I}-g_{h}\right) \\
& =\int_{\Omega} \sigma_{z}^{n+\lambda} \nabla\left(g-g_{h}\right) \cdot \nabla\left(g-g_{I}\right)+\int_{\Omega} \nabla\left(g-g_{h}\right) \cdot \nabla \psi-\int_{\Omega}\left(g_{I}-g_{h}\right) \nabla\left(\sigma_{z}^{n+\lambda}\right) \cdot \nabla\left(g-g_{h}\right) \\
& =\int_{\Omega} \sigma_{z}^{n+\lambda} \nabla\left(g-g_{h}\right) \cdot \nabla\left(g-g_{I}\right)+\int_{\Omega} \nabla\left(g-g_{h}\right) \cdot \nabla\left(\psi-\psi_{I}\right)-\int_{\Omega}\left(g_{I}-g_{h}\right) \nabla\left(\sigma_{z}^{n+\lambda}\right) \cdot \nabla\left(g-g_{h}\right) \\
& =\mathrm{I}+\mathrm{II}+\mathrm{III},
\end{aligned}
$$

where we used the Galerkin orthogonality of $g-g_{h}$. The third term is ommited in Lemma 5.1 of [21], but we shall see that this ommition is of no consequence. Indeed, applying Hölder inequality and Young inequality:

$$
\begin{aligned}
|\mathrm{III}| & \leq C \int_{\Omega} \sigma_{z}^{n+\lambda-1}\left|g_{I}-g_{h}\right|\left|\nabla\left(g-g_{h}\right)\right| \leq C\left(\int_{\Omega} \sigma_{z}^{n+\lambda}\left|\nabla\left(g-g_{h}\right)\right|^{2}\right)^{\frac{1}{2}}\left(\int_{\Omega} \sigma_{z}^{n+\lambda-1}\left|g_{I}-g_{h}\right|^{2}\right)^{\frac{1}{2}} \\
& \leq \frac{1}{4} \int_{\Omega} \sigma_{z}^{n+\lambda}\left|\nabla\left(g-g_{h}\right)\right|^{2}+C \int_{\Omega} \sigma_{z}^{n+\lambda-1}\left|g_{I}-g_{h}\right|^{2} .
\end{aligned}
$$

The first term can be kicked back to the left hand member. We will deal with the second term later. In a similar way, we can treat I, obtaining:

$$
\mathrm{I} \leq \frac{1}{4} \int_{\Omega} \sigma_{z}^{n+\lambda}\left|\nabla\left(g-g_{h}\right)\right|^{2}+C \int_{\Omega} \sigma_{z}^{n+\lambda}\left|\nabla\left(g-g_{I}\right)\right|^{2} .
$$

Again, the first term is kicked back to the left, whereas the second one is part of the desired estimate. Finally, for II we apply once again a combination of Hölder and Young inequalities obtaining:

$$
\mathrm{II} \leq \frac{1}{4} \int_{\Omega} \sigma_{z}^{n+\lambda}\left|\nabla\left(g-g_{h}\right)\right|^{2}+C \int_{\Omega} \sigma_{z}^{-n-\lambda}\left|\nabla\left(\psi-\psi_{I}\right)\right|^{2} .
$$

The first term is again kicked back to the left. We study the second one element by element and apply a standard interpolation result. $\nabla_{b} v$ denotes the derivatives of $v$ of order $b$ :

$$
\begin{aligned}
\int_{T} \sigma_{z}^{-n-\lambda}\left|\nabla\left(\psi-\psi_{I}\right)\right|^{2} & \leq \sigma_{z, T}^{-n-\lambda} \int_{T}\left|\nabla\left(\psi-\psi_{I}\right)\right|^{2} \leq C h_{T}^{2} \sigma_{z, T}^{-n-\lambda} \int_{T}\left|\nabla^{2} \psi\right|^{2} \\
& \leq\left. C h_{T}^{2} \int_{T} \sigma_{z}^{-n-\lambda} \sum_{0 \leq b \leq 1}\left|\nabla_{2-b} \sigma_{z}^{n+\lambda}\right|\right|^{2}\left|\nabla_{b}\left(g_{I}-g_{h}\right)\right|^{2} \\
& =C h_{T}^{2} \sum_{0 \leq b \leq 1} \int_{T} \sigma_{z}^{-n-\lambda+2(n+\lambda-2+b)}\left|\nabla_{b}\left(g_{I}-g_{h}\right)\right|^{2} \\
& \leq C h_{T}^{2} \sum_{0 \leq b \leq 1} \sigma_{z, T}^{n+\lambda-4+2 b} \int_{T}\left|\nabla_{b}\left(g_{I}-g_{h}\right)\right|^{2} .
\end{aligned}
$$

Observe that the summation takes only $b=0,1$, since $\left.\left(g_{I}-g_{h}\right)\right|_{T} \in \mathcal{P}_{1}$ and $\nabla^{2}\left(g_{I}-g_{h}\right)=0$. Now, we apply a well known inverse inequality and (4.4), obtaining:

$$
\begin{aligned}
\int_{T} \sigma_{z}^{-n-\lambda}\left|\nabla\left(\psi-\psi_{I}\right)\right|^{2} & \leq C h_{T}^{2-2 b} \sum_{0 \leq b \leq 1} \sigma_{z, T}^{n+\lambda-4+2 b} \int_{T}\left|g_{I}-g_{h}\right|^{2} \leq C \sum_{0 \leq b \leq 1} \sigma_{z, T}^{n+\lambda-4+2 b+2-2 b} \int_{T}\left|g_{I}-g_{h}\right|^{2} \\
& \leq C \int_{T} \sigma_{z}^{n+\lambda-2}\left|g_{I}-g_{h}\right|^{2}
\end{aligned}
$$


which is the same as the remainder of our estimate for III. The result follows by observing that:

$$
\int_{T} \sigma_{z}^{n+\lambda-2}\left|g_{I}-g_{h}\right|^{2} \leq C\left\{\int_{T} \sigma_{z}^{n+\lambda}\left|g-g_{I}\right|^{2}+\int_{T} \sigma_{z}^{n+\lambda}\left|g-g_{h}\right|^{2}\right\} .
$$

Lemma 4.6 is complemented by Lemma 4.8, that shows that the first term on the right hand side of (4.13) can be kicked back to the left. Combining these results, we obtain an estimate that depends only on the weighted error of the Lagrange interpolator, where a weighted interpolation error estimate (Lem. 4.7) can be applied.

The main idea is to introduce weighted norms with weights that resemble the graduation of the mesh. Hence, let us define:

$$
\rho(x)=\left(d(x)^{2}+\kappa^{2 m}\right)^{\frac{1}{2}},
$$

which is a smoothen distance to $x_{0}$. It is easy to check that:

$$
\left|D^{\gamma} \rho^{\lambda}(x)\right| \leq C \rho(x)^{\lambda-|\gamma|} .
$$

By definition of $L_{j}$ we have that

$$
\rho(x) \sim \rho_{j}:=\kappa^{m-j}, \quad \forall x \in L_{j} .
$$

We also denote $\rho_{T}$ an average value of $\rho$ over $T \in \mathcal{T}_{h}$, such that $\rho(x) \sim \rho_{T}$, for every $x \in T$.

Let us state a weighted interpolation result:

Lemma 4.7. For every $v \in H^{2}(\Omega)$ and $b=0,1$, denote $\nabla_{b} v$ the derivatives of order $b$ of $v$. Then, for every $\eta \in \mathbb{R}$, we have that:

$$
\int_{\Omega} \sigma_{z}^{\eta}(x)\left|\nabla_{b}\left(v-v_{I}\right)(x)\right|^{2} \mathrm{~d} x \leq C h^{2} \int_{\Omega} \sigma_{z}^{\eta+2-2 b}(x)\left|\rho(x)^{1-\mu} \nabla^{2} v(x)\right|^{2} \mathrm{~d} x .
$$

Proof. The result follows easily element-wise. We have, applying standard interpolation estimates, (4.11) and the graduation (4.1):

$$
\begin{aligned}
\int_{T} \sigma_{z}^{\eta}\left|\nabla_{b}\left(v-v_{I}\right)\right|^{2} & \leq \sigma_{T}^{\eta} \int_{T} \mid \nabla_{b}\left(v-v_{I}\right)^{2} \\
& \leq C h_{T}^{4-2 b} \sigma_{T}^{\eta} \int_{T}\left|\nabla^{2} v\right|^{2} \leq C h_{T}^{2} \sigma_{T}^{2-2 b} \sigma_{T}^{\eta} \int_{T}\left|\nabla^{2} v\right|^{2} \\
& \leq C h^{2} \rho_{T}^{2-2 \mu} \int_{T} \sigma_{z}^{\eta+2-2 b}(x)\left|\nabla^{2} v(x)\right|^{2} \mathrm{~d} x \\
& \leq C h^{2} \int_{T} \sigma_{z}^{\eta+2-2 b}(x)\left|\rho(x)^{1-\mu} \nabla^{2} v(x)\right|^{2} \mathrm{~d} x .
\end{aligned}
$$

The result follows summing up over all the elements of the triangulation.

Now, we can state Lemma 4.8. Since its proof is rather long, we split it in three parts. We present the main part here, but leave two technical lemmas for later.

Lemma 4.8. Being $\mathcal{T}_{h}$ a mesh graded according to (4.1), for every $\varepsilon>0$, there is some $\lambda>0$ and $t>1$ such that:

$$
\int_{\Omega} \sigma_{z}^{n+\lambda-2}\left(g-g_{h}\right)^{2} \leq \varepsilon \int \sigma_{z}^{n+\lambda}\left|\nabla\left(g-g_{h}\right)\right|^{2} .
$$


Proof. Let us define $v \in H_{0}^{1}(\Omega)$ such that:

$$
(\nabla v, \nabla w)=\left(\sigma_{z}^{n+\lambda-2}\left(g-g_{h}\right), w\right), \quad \forall w \in H_{0}^{1}(\Omega) .
$$

Hence, we have, thanks to Galerkin ortogonality, and Young's inequality:

$$
\begin{aligned}
\int_{\Omega} \sigma_{z}^{n+\lambda-2}\left|g-g_{h}\right|^{2} & =\int \nabla v \cdot \nabla\left(g-g_{h}\right)=\int \nabla\left(v-v_{I}\right) \cdot \nabla\left(g-g_{h}\right) \\
& \leq\left(\int_{\Omega} \sigma_{z}^{n+\lambda}\left|\nabla\left(g-g_{h}\right)\right|^{2}\right)^{\frac{1}{2}}\left(\int_{\Omega} \sigma_{z}^{-n-\lambda}\left|\nabla\left(v-v_{I}\right)\right|^{2}\right)^{\frac{1}{2}} \\
& \leq \frac{\varepsilon}{2} \int_{\Omega} \sigma_{z}^{n+\lambda}\left|\nabla\left(g-g_{h}\right)\right|^{2}+\frac{C}{\varepsilon} \int_{\Omega} \sigma_{z}^{-n-\lambda}\left|\nabla\left(v-v_{I}\right)\right|^{2}=A+B .
\end{aligned}
$$

$A$ is the desired estimate. For $B$, we can apply Lemma 4.7 with $\eta=-n-\lambda$ and $b=1$, obtaining:

$$
\int_{\Omega} \sigma_{z}^{-n-\lambda}\left|\nabla\left(v-v_{I}\right)\right|^{2} \leq C h^{2} \int_{\Omega} \sigma_{z}^{-n-\lambda}\left|\rho^{1-\mu} \nabla^{2} v\right|^{2} .
$$

Now, taking $q>\frac{n}{2}$ and $p$ its conjugate exponent, $\frac{1}{q}+\frac{1}{p}=1$ :

$$
\leq C h^{2} \underbrace{\left(\int_{\Omega}\left(\sigma_{z}^{-n-\lambda} \rho^{2-2 \mu}\right)^{q}\right)^{\frac{1}{q}}}_{D_{1}} \underbrace{\left\|\nabla^{2} v\right\|_{L^{2 p}(\Omega)}^{2}}_{D_{2}} .
$$

For $D_{2}$, we apply Gagliardo-Niremberg-Sobolev inequality with $r^{*}=2 p, r=\frac{2 n p}{n+2 p}$, and Hölder's inequality with exponents $s=\frac{2}{r}$ and $s^{\prime}=\frac{2}{2-r}$, and we obtain:

$$
\left\|\nabla^{2} v\right\|_{L^{2 p}(\Omega)}^{2} \leq C\left(\int_{\Omega}|\nabla \Delta v|^{r}\right)^{\frac{2}{r}} \leq C \underbrace{\left(\int_{\Omega} \sigma_{z}^{-n-\lambda+4}|\nabla \Delta v|^{2}\right)}_{D_{3}} \underbrace{\left(\int_{\Omega} \sigma_{z}^{(n+\lambda-4) \frac{s^{\prime}}{s}}\right)^{\frac{s}{s^{\prime}}}}_{D_{4}} .
$$

Observe that we need $r<2$, which implies $q>\frac{n}{2}$.

For $D_{3}$, recall that $\Delta v=\sigma_{z}^{n+\lambda-2}\left(g-g_{h}\right)$ which, along with (4.5), gives:

$$
|\nabla \Delta v|=\left|\nabla\left(\sigma_{z}^{n+\lambda-2}\left(g-g_{h}\right)\right)\right| \leq\left|\sigma_{z}^{n+\lambda-3}\left(g-g_{h}\right)\right|+\left|\sigma_{z}^{n+\lambda-2} \nabla\left(g-g_{h}\right)\right| .
$$

Hence:

$$
D_{3} \leq \int_{\Omega} \sigma_{z}^{n+\lambda-2}\left(g-g_{h}\right)^{2}+\int_{\Omega} \sigma_{z}^{n+\lambda}\left|\nabla\left(g-g_{h}\right)\right|^{2} .
$$

We have already stated that:

$$
\int_{\Omega} \sigma_{z}^{-n-\lambda}\left|\nabla\left(v-v_{I}\right)\right|^{2} \leq C h^{2} D_{1} D_{3} D_{4} .
$$

To bound $D_{1}$ and $D_{4}$ requieres some effort. Hence, we perform the task in separate lemmas, that are proven below. For now, let us just state that assuming $q$ is large enough so $n+(2-2 \mu-n-\lambda) q<0$ and that $\lambda<4-n s$ Lemmas 4.11 and 4.10 can be applied to $D_{1}$ and $D_{4}$ respectively, obtaining (recall that $\frac{1}{p}+\frac{1}{q}=1$ ):

$$
\int_{\Omega} \sigma_{z}^{-n-\lambda}\left|\nabla\left(v-v_{I}\right)\right|^{2} \leq C h^{2} h^{-2} h_{z}^{\frac{n}{q}-n-\lambda+2}\left(t h_{z}\right)^{\lambda-2+\frac{n}{p}} D_{3}=C t^{\lambda-2+\frac{n}{p}} D_{3} .
$$


Hence, for estimating the term $B$ in (4.15) we replace $D_{3}$ by (4.16), obtaining:

$$
\mathrm{II}=\frac{C}{\varepsilon} \int_{\Omega} \sigma_{z}^{-n-\lambda}\left|\nabla\left(v-v_{I}\right)\right|^{2} \leq \frac{C}{\varepsilon} t^{\lambda-2+\frac{n}{p}}\left\{\int_{\Omega} \sigma_{z}^{n+\lambda-2}\left(g-g_{h}\right)^{2}+\int_{\Omega} \sigma_{z}^{n+\lambda}\left|\nabla\left(g-g_{h}\right)\right|^{2}\right\} .
$$

Take into account that, since $s=\frac{n+2 p}{n p}$, the requirement $\lambda<4-n s$ is equivalent to $\lambda-2+\frac{n}{p}<0$. Consequently, the exponent of $t$ is negative, and we can fix a value of $t$ large enough so that $C t^{\lambda-2+\frac{n}{p}} \leq \frac{\varepsilon^{2}}{2}$. Hence:

$$
\int_{\Omega} \sigma_{z}^{n+\lambda-2}\left(g-g_{h}\right)^{2} \leq \varepsilon \int_{\Omega} \sigma_{z}^{n+\lambda}\left|\nabla\left(g-g_{h}\right)\right|^{2}+\frac{\varepsilon}{2} \int_{\Omega} \sigma_{z}^{n+\lambda-2}\left(g-g_{h}\right)^{2} .
$$

Finally, observe that we have imposed three conditions on $\lambda$ and $q: \lambda<2-\frac{n}{p}=2-n+\frac{n}{q}, \frac{n}{q}<2$ and $n+(2-2 \mu-n-\lambda) q<0$. Since it is possible to choose $\lambda>0$ and $q>2$ fullfilling all of them, te result follows.

Lemmas 4.6 and 4.8 give:

Corollory 4.9. Let $g_{I} \in V_{h}$ be the Lagrange interpolation of $g$, then it is possible to choose $\lambda>0$ and $t>1$ such that:

$$
\int_{\Omega} \sigma_{z}^{n+\lambda}\left|\nabla\left(g-g_{h}\right)\right|^{2} \leq C\left\{\int_{\Omega} \sigma_{z}^{n+\lambda-2}\left(g-g_{I}\right)^{2}+\int_{\Omega} \sigma_{z}^{n+\lambda}\left|\nabla\left(g-g_{I}\right)\right|^{2}\right\},
$$

for a constant $C$ independent of $z$.

Before completing the proof of Claim 1, we prove the results that were used in Lemma 4.8:

Lemma 4.10. Let $s>1, s^{\prime}$ such that $\frac{1}{s}+\frac{1}{s^{\prime}}=1$ and $\lambda>0$ such that $\lambda<4-n s$, then:

$$
\left(\int_{\Omega} \sigma_{z}(x)^{(n+\lambda-4) \frac{s^{\prime}}{s}} \mathrm{~d} x\right)^{\frac{s}{s^{\prime}}} \leq C\left(t h_{z}\right)^{\lambda-2+\frac{n}{p}}
$$

Proof.

$$
\begin{aligned}
\int_{\Omega} \sigma_{z}(x)^{(n+\lambda-4) \frac{s^{\prime}}{s}} \mathrm{~d} x & =\int_{\left\{|x-z| \leq t h_{z}\right\}} \sigma_{z}(x)^{(n+\lambda-4) \frac{s^{\prime}}{s}} \mathrm{~d} x+\int_{\left\{|x-z|>t h_{z}\right\}} \sigma_{z}(x)^{(n+\lambda-4) \frac{s^{\prime}}{s}} \mathrm{~d} x \\
& \leq \int_{\left\{|x-z| \leq t h_{z}\right\}}\left(t h_{z}\right)^{(n+\lambda-4) \frac{s^{\prime}}{s}} \mathrm{~d} x+\int_{\left\{|x-z|>t h_{z}\right\}}|x-z|^{(n+\lambda-4) \frac{s^{\prime}}{s}} \mathrm{~d} x \\
& \leq\left(t h_{z}\right)^{(n+\lambda-4) \frac{s^{\prime}}{s}+n}+\int_{\left\{|x-z|>t h_{z}\right\}}|x-z|^{(n+\lambda-4) \frac{s^{\prime}}{s}} \mathrm{~d} x .
\end{aligned}
$$

The second term can be estimated using spherical coordinates, taking $\xi=|x-z|$, and some fixed $R>\operatorname{diam}(\Omega)$, we have:

$$
\begin{aligned}
\int_{\left\{|x-z|>t h_{z}\right\}}|x-z|^{(n+\lambda-4) \frac{s^{\prime}}{s}} \mathrm{~d} x & =C \int_{t h_{z}}^{R} \xi^{(n+\lambda-4) \frac{s^{\prime}}{s}+n-1} d \xi \\
& =C\left(R^{(n+\lambda-4) \frac{s^{\prime}}{s}+n}-\left(t h_{z}\right)^{(n+\lambda-4) \frac{s^{\prime}}{s}+n}\right) .
\end{aligned}
$$

Assuming $(n+\lambda-4) \frac{s^{\prime}}{s}+n<0$ the second term dominates (for $h$ small enough), so we have:

$$
\left(\int_{\Omega} \sigma_{z}(x)^{(n+\lambda-4) \frac{s^{\prime}}{s}} \mathrm{~d} x\right)^{\frac{s}{s^{\prime}}} \leq C\left(t h_{z}\right)^{\left\{(n+\lambda-4) \frac{s^{\prime}}{s}+n\right\} \frac{s}{s^{\prime}}}=C\left(t h_{z}\right)^{(n+\lambda-4)+n \frac{s}{s^{\prime}}} .
$$

Observe that condition $(n+\lambda-4) \frac{s^{\prime}}{s}+n<0$ is equivalent to $n+\lambda-4+n \frac{s}{s^{\prime}}<0$ which, in turn, is equivalent to $\lambda<4-n s$. 
Lemma 4.11. Let $\eta>0$, and $q>1$ such that $n+(2-2 \mu-\eta) q<0$, then:

$$
\left(\int_{\Omega}\left(\sigma_{z}^{-\eta}(x) \rho^{2-2 \mu}(x)\right)^{q} \mathrm{~d} x\right)^{\frac{1}{q}} \leq C h^{-2} h_{z}^{\frac{n}{q}+2-\eta} .
$$

Proof. We center our analysis at the point $z$, which we assume to lie in the set $L_{\ell}$, and we use the decomposition (4.12) given by the sets $B_{z}, \Omega_{k}^{z}$ and $\Gamma_{i}^{z}$. For $x \in B_{z}$, we have:

$$
\int_{B_{z}}\left(\sigma_{z}(x)^{-\eta} \rho(x)^{2-2 \mu}\right)^{q} \mathrm{~d} x \leq\left(t h_{z}\right)^{-\eta q} \int_{B_{z}} \rho(x)^{(2-2 \mu) q} \mathrm{~d} x=\circledast .
$$

We consider two cases. If $x_{0} \in 2 B_{z}$, then $\rho(x) \leq C h_{z}$, so:

$$
\circledast \leq C\left(t h_{z}\right)^{-\eta q} h_{z}^{q(2-2 \mu)+n} \leq C h_{z}^{(2-\eta) q+n} h^{-2 q} .
$$

In the last step we used that $\eta>0, t>1$ and:

$$
h_{z}^{-2 q \mu}=h_{\ell}^{-2 q \mu}=\kappa^{(m-\ell(1-\mu))(-2 q \mu)}=2^{2 q m} 2^{2 q \ell(\mu-1)} \leq 2^{2 q m}=h^{-2 q} .
$$

On the other hand, if $x_{0} \notin 2 B_{z}$, then $\rho(x) \leq \rho(z)$, so:

$$
\begin{aligned}
\circledast & \leq C\left(t h_{z}\right)^{-\eta q} h_{z}^{n} \rho_{\ell}^{(2-2 \mu) q}=C t^{-\eta q} h_{z}^{-\eta q+n} h_{\ell}^{2 q} h^{-2 q} \\
& \leq C h_{z}^{(2-\eta) q+n} h^{-2 q},
\end{aligned}
$$

as in the previous case. We now consider the integral over $B_{z}^{c}$ separating $\cup_{j \leq \ell+1} L_{j}$ and $\cup_{j>\ell+1} L_{j}$.

Recall that on $L_{j}, \rho(x) \sim \rho_{j}=\kappa^{(m-j)}$. Taking into account that $\eta>0$ and $q>1$, we have:

$$
\begin{aligned}
\int_{B_{z}^{c} \cap\left(\cup_{j \leq \ell+1} L_{j}\right)}\left(\sigma_{z}(x)^{-\eta} \rho(x)^{2-2 \mu}\right)^{q} \mathrm{~d} x & \leq \sum_{j \leq \ell+1} \int_{B_{z}^{c} \cap L_{j}} \frac{\rho(x)^{(2-2 \mu) q}}{|x-z|^{\eta q}} \mathrm{~d} x \\
& \leq C \sum_{j \leq \ell+1} \rho_{j}^{(2-2 \mu) q} \sum_{k} \int_{B_{z}^{c} \cap L_{j} \cap \Omega_{k}^{z}} \frac{1}{|x-z|^{\eta q}} \mathrm{~d} x \\
& \leq C \sum_{j \leq \ell+1} \rho_{j}^{(2-2 \mu) q} \sum_{k}\left(2^{k} h_{z}\right)^{n-\eta q} \\
& \leq \sum_{j \leq \ell+1} \rho_{j}^{(2-2 \mu) q} h_{z}^{n-\eta q}=h_{z}^{n-\eta q} \sum_{j \leq \ell+1} \kappa^{(m-j)(2-2 \mu) q} \\
& \leq C h_{z}^{n-\eta q} \kappa^{(m-\ell)(2-2 \mu) q} .
\end{aligned}
$$

In order to finish the estimate, recall (4.9) and (4.8):

$$
=C h_{z}^{n-\eta q} \kappa^{[m-\ell(1-\mu)] 2 q} \kappa^{-m \mu 2 q}=C h_{z}^{(2-\eta) q+n} h^{-2 q} .
$$


Finally, we consider $B_{z}^{c} \cap\left(\cup_{j>\ell+1} L_{j}\right)$. We apply Lemma 4.5 with $\bar{y}=z$ :

$$
\begin{aligned}
\int_{B_{z}^{c} \cap_{j}>\ell+1}\left(\sigma_{z}(x)^{-\eta} \rho(x)^{2-2 \mu}\right)^{q} \mathrm{~d} x \leq \sum_{j>\ell+1} \int_{B_{z}^{c} \cap L_{j}} \frac{\rho(x)^{(2-2 \mu) q}}{|x-z|^{\eta q}} \mathrm{~d} x \\
\quad=\sum_{j>\ell+1} \rho_{j}^{(2-2 \mu) q} \sum_{k=\ell+\frac{1}{\mu}(j-\ell-1)}^{\ell+2+\frac{1}{\mu}(j-\ell)} \int_{B_{z}^{c} \cap L_{j} \cap \Omega_{k}^{z}} \frac{1}{\left(2^{k} h_{z}\right)^{\eta q}} \mathrm{~d} x \\
\leq C h_{z}^{n-\eta q} \sum_{j>\ell+1} \rho_{j}^{(2-2 \mu) q} \sum_{k=\ell+\frac{1}{\mu}(j-\ell-1)}^{\ell+\frac{1}{\mu}(j-\ell)} 2^{(n-\eta q) k} \\
\leq C h_{z}^{n-\eta q} \sum_{j>\ell+1} \rho_{j}^{(2-2 \mu) q} 2^{(n-\eta q)\left(\ell+\frac{1}{\mu}(j-\ell-1)\right)} \\
\quad=C h_{z}^{n-\eta q} \sum_{j>\ell+1} \kappa^{(m-j)(1-\mu) 2 q} \kappa^{(n-\eta q)(-\mu \ell-j+\ell+1)} \\
\leq C h_{z}^{n-\eta q} \kappa^{m(1-\mu) 2 q+(n-\eta q) \ell(1-\mu)} \kappa^{n-\eta q} \sum_{j>\ell+1} \kappa^{[-(1-\mu) 2 q-(n-\eta q)] j}=\odot .
\end{aligned}
$$

The factor $\kappa^{n-\eta q}$ can be absorbed in the constant $C$. Observe that this depends on $n, \eta, q$ and $\mu_{0}$, but not on $m$, so this does not impose any restrictions on the mesh size parameter $h$. In order to complete the proof, we recall that by hypothesis $-(1-\mu) 2 q-(n-\eta q)=-n-(2-2 \mu-\eta) q>0$, so:

$$
\begin{aligned}
\odot & \leq C h_{z}^{n-\eta q} \kappa^{m(1-\mu) 2 q+(n-\eta q) \ell(1-\mu)} \kappa^{[-(1-\mu) 2 q-(n-\eta q)] \ell} \\
& \leq C h_{z}^{n-\eta q} \kappa^{[m-\ell(1-\mu)] 2 q} \kappa^{-m \mu 2 q} \kappa^{-\mu(n-\eta q)} \\
& \leq C h_{z}^{n-\eta q} h_{z}^{2 q} h^{-2 q} \leq C h^{-2 q} h_{z}^{n+(2-\eta) q} .
\end{aligned}
$$

And the proof is complete.

Finally, we can prove Claim 1:

Proof of Claim 1 for graded meshes. We have, applying Corollary 4.9 and Lemma 4.7:

$$
\begin{aligned}
M^{2} & :=\left\|h_{z}^{-\frac{\lambda}{2}} \sigma_{z}^{\frac{n+\lambda}{2}} \nabla\left(g-g_{h}\right)\right\|_{L^{2}(\Omega)}^{2} \leq h_{z}^{-\lambda}\left\{\int_{\Omega} \sigma_{z}^{n+\lambda-2}\left(g-g_{I}\right)^{2}+\int_{\Omega} \sigma_{z}^{n+\lambda}\left|\nabla\left(g-g_{I}\right)\right|^{2}\right\} \\
& \leq C h_{z}^{-\lambda} h^{2} \underbrace{\int_{\Omega} \rho^{2-2 \mu}\left|\sigma_{z}^{\frac{n+\lambda}{2}} \nabla_{2} g\right|^{2}}_{E} .
\end{aligned}
$$

Moreover:

$$
E \leq\{\underbrace{\int_{\Omega} \rho^{2-2 \mu}\left|\nabla_{2}\left(\sigma_{z}^{\frac{n+\lambda}{2}} g\right)\right|^{2}}_{\text {I }}+\underbrace{\int_{\Omega} \rho^{2-2 \mu} \sigma_{z}^{n+\lambda-2}|\nabla g|^{2}}_{\text {II }}+\underbrace{\int_{\Omega} \rho^{2-2 \mu} \sigma_{z}^{n+\lambda-4} g^{2}}_{\text {III }}\} .
$$

Since $\rho^{2(1-\mu)} \in A_{2}(\Omega)$, we can apply Theorem 3.1 to I:

$$
\mathrm{I} \leq \int_{\Omega} \rho^{2-2 \mu}\left|\Delta\left(\sigma_{z}^{\frac{n+\lambda}{2}} g\right)\right|^{2} \leq \mathrm{II}+\mathrm{III}+\underbrace{\int_{\Omega} \rho^{2-2 \mu} \sigma_{z}^{n+\lambda}\left|\partial \eta_{z}\right|^{2}}_{\mathrm{IV}} .
$$


Following a standard procedure, we prove that the term II can be avoided, obtaining $M^{2} \leq C(\mathrm{III}+\mathrm{IV})$ :

$$
\begin{aligned}
\mathrm{II} & \leq \int_{\Omega} \nabla g \cdot \nabla\left(\rho^{2-2 \mu} \sigma_{z}^{n+\lambda-2} g\right)+\int_{\Omega} \nabla g \cdot \nabla\left(\rho^{2-2 \mu}\right) \sigma_{z}^{n+\lambda-2} g+\int_{\Omega}|\nabla g| \rho^{2-2 \mu} \sigma_{z}^{n+\lambda-3} g \\
& \leq \int_{\Omega} \rho^{2-2 \mu} \sigma_{z}^{n+\lambda-2} g \partial \eta_{z}+\int_{\Omega}|\nabla g| \rho^{1-2 \mu} \sigma_{z}^{n+\lambda-2} g+\int_{\Omega}|\nabla g| \rho^{2-2 \mu} \sigma_{z}^{n+\lambda-3} g .
\end{aligned}
$$

Applying Hölder's inequality in each term:

$$
\begin{aligned}
\leq & \left(\int_{\Omega} \rho^{2-2 \mu} \sigma_{z}^{n+\lambda-4} g^{2}\right)^{\frac{1}{2}}\left(\int_{\Omega} \rho^{2-2 \mu} \sigma_{z}^{n+\lambda}\left|\partial \delta_{z}\right|^{2}\right)^{\frac{1}{2}} \\
& +\left(\int_{\Omega} \rho^{2-2 \mu} \sigma_{z}^{n+\lambda-4} g^{2}\right)^{\frac{1}{2}}\left(\int_{\Omega} \rho^{-2 \mu} \sigma_{z}^{n+\lambda}|\nabla g|^{2}\right)^{\frac{1}{2}} \\
& +\left(\int_{\Omega} \rho^{2-2 \mu} \sigma_{z}^{n+\lambda-4} g^{2}\right)^{\frac{1}{2}}\left(\int_{\Omega} \rho^{2-2 \mu} \sigma_{z}^{n+\lambda-2}|\nabla g|^{2}\right)^{\frac{1}{2}} .
\end{aligned}
$$

And then, Young's inequality leads to:

$$
\leq C \mathrm{III}+\varepsilon \mathrm{IV}+\varepsilon \underbrace{\int_{\Omega} \rho^{-2 \mu}\left|\sigma_{z}^{\frac{n+\lambda}{2}} \nabla g\right|^{2}}_{\mathrm{V}}+\varepsilon \mathrm{II} .
$$

For $V$ we apply Theorem 2.2 with $\tau_{1}=\tau_{2}=2 \gamma_{2}=-\mu$ and $\gamma_{2}=1-\mu$, which gives:

$$
\begin{aligned}
V & \leq C \int_{\Omega}\left|x-x_{0}\right|^{-2 \mu}\left|\sigma_{z}^{\frac{n+\lambda}{2}} \nabla g\right|^{2} \leq C \int_{\Omega}\left|x-x_{0}\right|^{2-2 \mu}\left|\nabla\left(\sigma_{z}^{\frac{n+\lambda}{2}} \nabla g\right)\right|^{2} \\
& \leq \int_{\Omega} \rho^{2-2 \mu}\left|\nabla\left(\sigma_{z^{2}}^{\frac{n+\lambda}{2}} \nabla g\right)\right|^{2} \\
& \leq C \int_{\Omega} \rho^{2-2 \mu} \sigma_{z}^{n+\lambda-2}|\nabla g|^{2}+C \int_{\Omega} \rho^{2-2 \mu}\left|\sigma_{z^{\frac{n+\lambda}{2}}}^{n+\lambda} \nabla_{2}\right|^{2}=C(\mathrm{II}+E) .
\end{aligned}
$$

Replacing this in the previous estimate we have:

$$
\mathrm{II} \leq C \mathrm{III}+\varepsilon \mathrm{IV}+\varepsilon(1+C) \mathrm{II}+C \varepsilon E,
$$

which in turn, taking $\varepsilon$ small enough implies:

$$
\mathrm{II} \leq C \mathrm{III}+\varepsilon \mathrm{IV}+C \varepsilon E .
$$

Replacing in the estimate for $E$, we have:

$$
E \leq C(\mathrm{III}+\mathrm{IV})
$$

as desired. IV is easily estimated by:

$$
\mathrm{IV} \leq \int_{T_{z}} \rho^{2-2 \mu} \sigma_{z}^{n+\lambda}\left|\partial \eta_{z}\right|^{2} \leq \rho_{z}^{2(1-\mu)} h_{z}^{n+\lambda-2 n-2} h_{z}^{n} \leq h^{-2} h_{z}^{2+\lambda-n-2+n}=h^{-2} h_{z}^{\lambda} .
$$

III, specially in the case $n=3$, requires some effort. We begin applying Hölder's inequality:

$$
\mathrm{III} \leq\left(\int_{\Omega}\left(\rho^{2-2 \mu} \sigma_{z}^{-2+\lambda}\right)^{q}\right)^{\frac{1}{q}}\left(\int_{\Omega} \sigma_{z}^{(n-2) p} g^{2 p}\right)^{\frac{1}{p}} .
$$


Observe that if $n=2$, the exponent of the weight $\sigma_{z}$ in the second factor vanishes, which simplifies the estimate. The first factor is estimated in Lemma 4.11, taking $\eta=2-\lambda$, which gives:

$$
\mathrm{III} \leq h^{-2} h_{z}^{\frac{n}{q}+\lambda}\left(\int_{\Omega} \sigma_{z}^{(n-2) p} g^{2 p}\right)^{\frac{1}{p}},
$$

provided that $\lambda<2 \mu$. Now, for the remaining factor we consider the more difficult case $n=3$, where Theorem 2.2 is needed. We have that:

$$
\left(\int_{\Omega} \sigma_{z}^{(n-2) p} g^{2 p}\right)^{\frac{1}{p}}=\left\|\sigma_{z^{\frac{n-2}{2}}} g\right\|_{L^{2 p}(\Omega)}^{2}
$$

Taking $y \in H_{0}^{1}(\Omega)$ the solution of the problem:

$$
(\nabla y, \nabla \varphi)=\left(\operatorname{sign}(g) g^{2 p-1} \sigma_{z}^{(n-2) p}, \varphi\right) \quad \forall \varphi \in H_{0}^{1}(\Omega) .
$$

Hence:

$$
\begin{aligned}
\left\|\sigma_{z}^{\frac{n-2}{2}} g\right\|_{L^{2 p}(\Omega)}^{2 p} & =(\nabla y, \nabla g)=\left(y, \partial \eta_{z}\right)=\left(-\partial y, \eta_{z}\right) \\
& \leq\left\|\sigma_{z}^{\gamma} \nabla y\right\|_{L^{s}(\Omega)}\left\|\eta_{z} \sigma_{z}^{-\gamma}\right\|_{L^{s^{\prime}}\left(T_{z}\right)} \leq h_{z}^{-n+\frac{n}{s^{\prime}}-\gamma}\left\|\sigma_{z}^{\gamma} \partial y\right\|_{L^{s}(\Omega)} \\
& =h_{z}^{-\gamma-\frac{n}{s}}\left\|\sigma_{z}^{\gamma} \partial y\right\|_{L^{s}(\Omega)}=\star,
\end{aligned}
$$

where $\gamma$ and $s$ are determined below. Now, we apply Theorem 2.2, with exponents $\tau_{1}=s$ and $\tau_{2}=r, \gamma_{1}=\gamma$ and $\gamma_{2}=\alpha$. It is easy to check that the inequality can be applied to the smoothen weight $\sigma_{z}$ instead of $|x-z|$ :

$$
\star \leq h_{z}^{-\gamma-\frac{n}{s}}\left\|\sigma_{z}^{\alpha} \nabla_{2} y\right\|_{L^{r}(\Omega)} .
$$

We choose $\alpha=-\frac{n-2}{2}$ and $r=\frac{2 p}{2 p-1}$ and observe that $\sigma_{z}^{-\frac{n-2}{2} r} \in A_{r}(\Omega)$. Moreover, we can take $p \searrow 1$, so $r \nearrow 2$. Consequently, we can apply Theorem 3.1, obtaining:

$$
\begin{aligned}
\star & \leq h_{z}^{-\gamma-\frac{n}{s}}\left\|\sigma_{z}^{\alpha} \Delta y\right\|_{L^{r}(\Omega)} \leq h_{z}^{-\gamma-\frac{n}{s}}\left\|\sigma_{z}^{-\frac{n-2}{2}+\frac{n-2}{2} 2 p} g^{2 p-1}\right\|_{L^{r}(\Omega)} \\
& =h_{z}^{-\gamma-\frac{n}{s}}\left\|\sigma_{z}^{\frac{n-2}{2}} g\right\|_{L^{2 p}(\Omega)}^{2 p-1} .
\end{aligned}
$$

Hence, we can kick back the last factor, and apply condition (2.4), obtaining:

$$
\left\|\sigma_{z}^{\frac{n-2}{2}} g\right\|^{2} \leq C h_{z}^{-2 \gamma-\frac{2 n}{s}} \leq C h_{z}^{-\frac{2 n}{r}-2(\alpha-1)}=C h_{z}^{-n \frac{2 p-1}{p}+n}=C h_{z}^{-n+\frac{n}{p}} .
$$

This implies the same estimate than the one obtained for IV:

$$
\mathrm{III} \leq C h^{-2} h_{z}^{\lambda},
$$

which gives the desired result.

The case $n=2$ is a little simpler: since the weight $\sigma_{z}$ does not appear in the $L^{2 p}$ norm of $g$, we can take $\gamma=0$ and apply Sobolev inequality, instead of Theorem 2.2. The reader can follow the details in Lemma 4.4 of [21] or in Lemma 8.3.7 of [7].

Observe that the proof is independent of the weight $\omega$ considered. Applying Theorem 4.3 with $\omega=d^{2 \alpha}$ we obtain Theorem 4.2 . 
Remark 4.12. Our proof of Theorem 4.3 holds for meshes graded according to (4.1) towards any point in $\bar{\Omega}$. Indeed, Theorem 3.1 is used in the proof of Claim 1 for the regularized weight $\rho^{2(1-\mu)}$, that belongs to $A_{2}(\Omega)$ for every $\mu>\mu_{0}$ regardless of the position of $x_{0}$. Hence, Theorem 4.3 can be applied to a variety of problems, where graded meshes are used. Moreover, since $\omega \equiv 1$ belongs to $A_{1}$, Corollary 4.4 provides an unweighted stability result for $u \in W^{1, p}(\Omega), 2<p<\infty$. As an example of a possible interesting application, we can mention elliptic problems on convex polygons with meshes that are locally graded toward the vertices, such as the ones considered in [21].

\section{A priori ERROR ESTIMATES}

The stability of the Galerkin projection (Thm. 4.2) along with the a priori estimate for the continuous problem (Thm. 2.1), lead to the following well-posedness result for the discrete problem:

Theorem 5.1. Let $\Omega \subset \mathbb{R}^{n}$ be $\mathcal{C}^{1,1}$ domain or a convex polygon or polyhedron, $x_{0} \in \Omega,-\frac{n}{2}<\alpha<\frac{n}{2}$, $f \in\left(H_{0,-\alpha}^{1}\right)^{\prime}$ and $u \in H_{0, \alpha}^{1}(\Omega)$ the unique solution of problem (2.3). Also, let $\mathcal{T}_{h}$ be a graded mesh with graduation parameter $\mu$, defined as in (4.1), and $V_{h}=V_{h}^{1}$ the space of piecewise polynomials of degree 1 . Then, the problem of finding $u_{h} \in V_{h}$ such that:

$$
\left(\nabla u_{h}, \nabla \varphi\right)=(f, \varphi) \quad \forall \varphi \in V_{h},
$$

admits a unique solution that satisfies the best approximation property (4.3).

In this section, we give a priori estimates in $H_{0, \alpha}^{1}$ and $L_{\beta}^{2}$ for $u-u_{h}$. The estimates are based on two main assumptions. On the one hand, we take:

$$
-\frac{n}{2}+1<\alpha<\frac{n}{2}
$$

which implies $H_{0, \alpha}^{1} \subset K_{\alpha-1}^{1}$. In order to avoid confusions, we denote $\alpha$ the exponent of the weight needed for $\nabla u$. Hence, we have that $u \in H_{\alpha}^{1}$ and $u \in L_{\alpha-1}^{2}$. On the other hand, we need some regularity for the second derivatives of $u$, so we assume that $u \in K_{\alpha-1}^{2}$.

It is important to emphasize that (5.1) (as well as the assumption that $u \in K_{\alpha-1}^{2}$ ) is a general condition necessary for our error analysis. However all our results are based on the fact that the right hand side $f$ belongs to $\left(H_{0,-\alpha}^{1}(\Omega)\right)^{\prime}$, which may impose further restrictions on $\alpha$. Indeed, we can state the following corollaries concerning Examples 2.3 and 2.4 respectively. Observe that in both cases $u \in K_{\alpha-1}^{2}(\Omega)$, but the conditions on $\alpha$ are more restrictive than (5.1):

Corollory 5.2. If $\frac{n}{2}-1<\alpha<\frac{n}{2}$, Theorem 5.1 holds for (2.3) with $f=\delta_{x_{0}}$.

Corollory 5.3. In $n=3$, taking $s-\frac{5}{2}<\alpha<\frac{3}{2}$, Theorem 5.1 holds for $(2.3)$ with $f(x)=d(x)^{-s}$.

From now on, $u$ is the continuous solution of $(2.3)$ and $u_{h}$ its Galerkin projection on a mesh $\mathcal{T}_{h}$ graded according to (4.1). We state our error estimates for $u-u_{h}$ in this general setting, but in complementary remarks we show how these estimates apply to the most interesting example given by $f=\delta_{x_{0}}$.

For simplicity, our estimates are given in terms of the mesh parameter $h$, but thanks to Remark 4.1, this is equivalent to state them in terms of the numbers of degrees of freedom.

Since (4.3) holds, it is enough to give error estimates for an appropriate interpolation operator $I_{h}: K_{\alpha-1}^{2} \rightarrow V_{h}$. Following [10] we define $\mathcal{T}_{h}^{\text {near }}=\left\{T \in \mathcal{T}_{h}: x_{0} \in \bar{T}\right\}$ and $\mathcal{T}_{h}^{\text {far }}=\mathcal{T} \backslash \mathcal{T}_{h}^{\text {near }}$. We frecuently abuse notation, writing $\mathcal{T}_{h}^{\text {near,far }}$ for denoting $\cup_{T \in \mathcal{T}_{h}^{\text {near,far }}} T$.

We also consider the patches:

$$
S_{T}=\bigcup_{T^{\prime} \cap T \neq \emptyset} T^{\prime} \quad \text { and } \quad S_{T}^{\prime}=\bigcup_{T^{\prime} \in \mathcal{T}_{h}^{\text {near }}, T^{\prime} \cap T \neq \emptyset} T^{\prime} .
$$


Let $\left\{x_{i}\right\}_{i=1}^{N_{h}}$ be the set of nodes of $\mathcal{T}_{h}$, and $\left\{\phi_{i}\right\}$ the nodal basis: $\left.\phi_{i}\right|_{T} \in \mathcal{P}_{1}, \phi_{i}\left(x_{j}\right)=\delta_{i j}$. We define:

$$
I_{h} u(x)=\sum_{i=1}^{N_{h}} a_{i} \phi_{i}(x) .
$$

For nodes $x_{i} \in \mathcal{T}_{h}^{\text {far }}, I_{h}$ is the Lagrange interpolator, given by $a_{i}=u\left(x_{i}\right)$. This definition is allowed by the fact that functions in $K_{\alpha-1}^{2}$ belong to $H^{2}\left(\mathcal{T}_{h}^{\text {far }}\right)$. On the other hand, for nodes $x_{i} \in \mathcal{T}_{h}^{\text {near }}$ we take $a_{i}=0$.

The following lemma in stated in Lemma 3.5 of [10].

Lemma 5.4. Let $u \in K_{\alpha-1}^{2}$, then $I_{h} u$ satisfies:

$$
\begin{array}{rlrl}
\left|u-I_{h} u\right|_{H^{\ell}(T)} & \leq C h_{T}^{2-\ell}|u|_{H^{2}(T)}, & & \text { if } T \in \mathcal{T}_{h}^{\mathrm{far}}, \ell=0,1 \\
\left|I_{h} u\right|_{H_{\alpha}^{1}(T)} & \leq C_{\alpha}\|u\|_{K_{\alpha-1}^{2}\left(S_{T}^{\prime}\right)}, \quad \text { if } T \in \mathcal{T}_{h}^{\text {near }},
\end{array}
$$

for every $u \in K_{\alpha-1}^{2}$ and $\ell=0,1$. Moreover, $C_{\alpha} \sim(2 \alpha+n)^{-\frac{1}{2}}$.

Proof. Equation (5.3) is a well known result, valid since $u \in H^{2}(T)$ for $T \in \mathcal{T}^{\text {far }}$. On the other hand, for (5.4), take $D_{T}=\left\{i: \bar{T}_{i} \cap \bar{T} \neq \emptyset, T_{i} \subset \mathcal{T}^{\text {far }}\right\}$. Then:

$$
\left|I_{h} u\right|_{H_{\alpha}^{1}(T)} \leq \sum_{i \in D_{T}} \underbrace{\left|a_{i}\right|}_{A} \underbrace{\left|\phi_{i}\right|_{H_{\alpha}^{1}(T)}}_{B} .
$$

Observe that $\left|\nabla \phi_{i}\right| \sim h_{T}^{-1}$ and using polar or spherical coordinates we have that $\int_{T} r(x)^{2 \alpha} \mathrm{d} x \leq|T| h_{T}^{2 \alpha}$, so $B \leq C|T|^{\frac{1}{2}} h_{T}^{\alpha-1}$. On the other hand, take $\widehat{T}$ the reference element with vertices on $\left\{x_{0}\right\}$, and the canonical vectors $e^{i}$ such that $\left(e^{i}\right)_{j}=\delta_{i, j}$, and consider the map $F_{T}: \widehat{T} \rightarrow T$ such that $F_{T}(\widehat{x})=x$. We define $\widehat{v}(\widehat{x})=v(x)$. Observe that: $\widehat{\nabla} \widehat{u} \sim h_{T} \nabla u$. Then:

$$
\begin{aligned}
\left|a_{i}\right| & =\left|u\left(x_{i}\right)\right| \leq\|u\|_{L^{\infty}\left(T_{i}\right)}=\|\hat{u}\|_{L^{\infty}(\hat{T})} \leq C\|\hat{u}\|_{H^{2}(\hat{T})}=C\left(\sum_{j=0}^{2}|\hat{u}|_{H^{j}(\hat{T})}^{2}\right)^{\frac{1}{2}} \\
& =C|T|^{-\frac{1}{2}}\left(\sum_{j=0}^{2} h_{T}^{2 j}|u|_{H^{j}(T)}^{2}\right)^{\frac{1}{2}} \leq C|T|^{-\frac{1}{2}}\left(\sum_{j=0}^{2} h_{T}^{2 j} h_{T}^{2-2 \alpha-2 j}|u|_{K_{\alpha-1}^{j}(T)}^{2}\right)^{\frac{1}{2}} \\
& \leq C|T|^{-\frac{1}{2}}\left(\sum_{j=0}^{2} h_{T}^{2-2 \alpha}|u|_{K_{\alpha-1}^{j}(T)}^{2}\right)^{\frac{1}{2}}
\end{aligned}
$$

and joining the estimates for $A$ and $B$ the result follows.

Theorem 5.5. Let $\eta<\alpha-1$ be such that $u \in K_{\eta}^{2}$. Then, for $\mu \leq \alpha-1-\eta$, we have:

$$
\left|u-u_{h}\right|_{H_{\alpha}^{1}(\Omega)} \leq C h\|u\|_{K_{\eta}^{2}(\Omega)} .
$$

Proof. It is enough to take $I_{h} u$ instead of $u_{h}$. We prove the result elementwise. For every $T$ such that $r_{T}>1$, the result follows directly from (5.3). For $T \in \mathcal{T}_{h}^{\text {far }}$, such that $r_{T} \leq 1$ :

$$
\begin{aligned}
\left|u-I_{h} u\right|_{H_{\alpha}^{1}(T)} & \leq C r_{T}^{\alpha}\left|u-I_{h} u\right|_{H^{1}(T)} \leq C r_{T}^{\alpha} h_{T}|u|_{H^{2}(T)} \\
& \leq C r_{T}^{\alpha} h_{T} r_{T}^{-\eta-2}|u|_{K_{\eta}^{2}(T)} \leq C r_{T}^{\alpha-\eta-2} h r_{T}^{1-\mu}|u|_{K_{\eta}^{2}(T)} \\
& \leq h r_{T}^{\alpha-1-\eta-\mu}|u|_{K_{\eta}^{2}(T)} \leq C h|u|_{K_{\eta}^{2}(T)} .
\end{aligned}
$$


We have used that $r_{T} \sim \max _{x \in T}\left|x-x_{0}\right|$.

Now, for $T \in \mathcal{T}_{h}^{\text {near }}$, thanks to (5.4) we have:

$$
\begin{aligned}
\left|u-I_{h} u\right|_{H_{\alpha}^{1}(T)} & \leq|u|_{H_{\alpha}^{1}(T)}+\left|I_{h} u\right|_{H_{\alpha}^{1}(T)} \\
& \leq C r_{T}^{\alpha-1-\eta}|u|_{K_{\eta}^{1}(T)}+C_{\alpha}\|u\|_{K_{\alpha-1}^{2}\left(S_{T}^{\prime}\right)} \\
& \leq h^{\frac{\alpha-1-\eta}{\mu}}|u|_{K_{\eta}^{1}(T)}+C_{\alpha} h^{\frac{\alpha-1-\eta}{\mu}}\|u\|_{K_{\eta}^{2}\left(S_{T}^{\prime}\right)} \leq C h\|u\|_{K_{\eta}^{2}\left(S_{T}\right)} .
\end{aligned}
$$

Corollory 5.6. Let $\eta<\alpha-1$ be such that $u \in K_{\eta}^{2}$, then for $\mu>\alpha-1-\eta$, we have:

$$
\left|u-I_{h} u\right|_{H_{\alpha}^{1}(\Omega)} \leq C_{\alpha} h^{\frac{\alpha-1-\eta}{\mu}}\|u\|_{K_{\eta}^{2}(\Omega)} .
$$

Proof. For $T \in \mathcal{T}_{h}^{\text {near }}$ this prove is included in the previous Theorem. For $T \in \mathcal{T}_{h}^{\text {far }}$, it is enough to observe that $r_{T}^{\alpha-1-\eta-\mu} \leq h^{\frac{\alpha-1-\eta-\mu}{\mu}}$.

Remark 5.7. For $f=\delta_{x_{0}}$, we have that $u \in K_{\eta}^{2}(\Omega)$ for every $\eta>\frac{n}{2}-2$. Inserting this in Theorem 5.5 we have that $\mu \leq \alpha-1-\eta<\alpha-1-\left(\frac{n}{2}-2\right)=\alpha+1-\frac{n}{2}$. Hence, taking

$$
\mu<\alpha+1-\frac{n}{2}
$$

we have that:

$$
\left\|\nabla\left(u-u_{h}\right)\right\|_{L_{\alpha}^{2}(\Omega)}=C h\|u\|_{K_{\eta}^{2}(\Omega)} .
$$

Recall condition (2.6), that says that $\alpha>\frac{n}{2}-1$. Now, if $\alpha \rightarrow \frac{n}{2}-1, \mu \rightarrow 0$, which invalidates our analysis.

So far, we have recovered optimal rates of converge for the gradient of the solution in weighted spaces, using graded meshes. Now, we want to use this result for obtaining optimal rates of converce in $L_{\beta}^{2}$. We do this through an Aubin-Nistche argument in weighted spaces.

Take $f_{\beta}=d^{2 \beta}\left|u-u_{h}\right|$. Then: $\left\|f_{\beta}\right\|_{L_{-\beta}^{2}(\Omega)}=\left\|u-u_{h}\right\|_{L_{\beta}^{2}(\Omega)}$. Let $w_{\beta} \in H_{-\alpha}^{1}$ be such that:

$$
\left(\nabla w_{\beta}, \nabla \varphi\right)=\left(f_{\beta}, \varphi\right) \quad \forall \varphi \in H_{0, \alpha}^{1} .
$$

Observe that $f_{\beta} \in L_{-\beta}^{2} \subset L_{1-\alpha}^{2}=\left(L_{\alpha-1}^{2}\right)^{\prime}$, provided that $\alpha<1+\beta$. On the other hand, $H_{0, \alpha}^{1}(\Omega) \subset L_{\alpha-1}^{2}(\Omega)$ (since (5.1) holds), so $f_{\beta}$ defines a bounded operator on $H_{0, \alpha}^{1}$. This implies that (5.6) with $\alpha<1+\beta$ admits a unique solution $w_{\beta} \in H_{0,-\alpha}^{1}$.

Thence, we have, taking $w_{\beta, h} \in V_{h}$ the Galerkin projection of $w_{\beta}, \eta$ such that $u \in K_{\eta}^{2}$ and $\mu \leq \alpha-1-\eta$ :

$$
\begin{aligned}
\left\|u-u_{h}\right\|_{L_{\beta}^{2}(\Omega)}^{2} & =\left(u-u_{h}, f_{\beta}\right)=\left(\nabla\left(u-u_{h}\right), \nabla w_{\beta}\right) \\
& =\left(\nabla\left(u-u_{h}\right), \nabla\left(w_{\beta}-w_{\beta, h}\right)\right) \\
& \leq\left\|\nabla\left(u-u_{h}\right)\right\|_{L_{\alpha}^{2}(\Omega)}\left\|\nabla\left(w_{\beta}-w_{\beta, h}\right)\right\|_{L_{-\alpha}^{2}(\Omega)} \\
& \leq C h\left\|\nabla\left(w_{\beta}-w_{\beta, h}\right)\right\|_{L_{-\alpha}^{2}(\Omega)}\|u\|_{K_{\eta}^{2}(\Omega)} .
\end{aligned}
$$

Now, we need to study the approximation error for $w_{\beta}$ in $H_{-\alpha}^{1}$. Some effort is needed due to the negative weight. We begin proving a local Poincaré inequality:

Lemma 5.8 (Local Poincaré inequality). Let $T$ be such that $x_{0}$ is one of its vertices, and take $v \in H_{-\alpha}^{1}(T)$, for $\alpha$ satisfying (5.1) such that $\int_{T} v=0$. Then:

$$
\|v\|_{L_{-\alpha}^{2}(T)} \leq C h_{T}^{1-\alpha+\beta}\|\nabla v\|_{L_{-\beta}^{2}(T)}
$$


holds for every $\beta \geq \alpha-1$, with a constant $C$ independent of $T$ and $v$.

Proof. As in Lemma 5.4, we denote $\widehat{T}$ the reference element and $F_{T}: \widehat{T} \rightarrow T, F_{T}(\widehat{x})=x$. mapping $\widehat{T}$ in $T$. We define $\widehat{v}(\widehat{x})=v(x)$. Observe that the distance $\widehat{r}$ in $\widehat{T}$ satisfies: $h_{T} \widehat{r}(\widehat{x}) \sim r\left(F_{T}(\widehat{x})\right)$. Hence, we have:

$$
\|v\|_{L_{-\alpha}^{2}(T)}=\left(\int_{T} v(x)^{2} r(x)^{-2 \alpha} \mathrm{d} x\right)^{\frac{1}{2}} \sim h_{T}^{-\alpha} J_{T}^{\frac{1}{2}}\left(\int_{\widehat{T}} \widehat{v}(\widehat{x}) \widehat{r}(\widehat{x})^{-2 \alpha} d \widehat{x}\right)^{\frac{1}{2}}=I
$$

where $J_{T}=\left|\operatorname{det}\left(D F_{T}\right)\right|$. Now, we apply Theorem 2.2 with $\tau_{1}=\tau_{2}=2, \gamma_{1}=-\alpha$ and $\gamma_{2}=1-\alpha$ on $\widehat{T}$ and go back to $T$, taking into account that $\widehat{\nabla} \widehat{v} \sim h_{T} \nabla v$ :

$$
\begin{aligned}
I & \lesssim h_{T}^{-\alpha} J_{T}^{\frac{1}{2}}\left(\int_{\widehat{T}}|\widehat{\nabla} \widehat{v}|^{2} \widehat{r}(\widehat{x})^{2(1-\alpha)} \mathrm{d} \widehat{x}\right)^{\frac{1}{2}} \\
& \leq C h_{T}^{-\alpha}\left(\int_{T} h_{T}^{2}|\nabla v|^{2} h_{T}^{2(\alpha-1)} r(x)^{2(1-\alpha)} \mathrm{d} x\right)^{\frac{1}{2}} \\
& \leq C\|\nabla v\|_{L_{1-\alpha}^{2}(T)} \leq C h_{T}^{1+\beta-\alpha}\|\nabla v\|_{L_{-\beta}^{2}(T)} .
\end{aligned}
$$

Observe that $\beta>-\frac{n}{2}$, so $f_{\beta} \in\left(H_{0}^{1}\right)^{\prime}$ and, consequently $w_{\beta} \in H^{2}$. This implies that the standard Lagrange interpolator can be used for interpolating $w_{\beta}$. Hence, we take $\Pi_{h}(v)$ defined as in $(5.2)$, but with $a_{i}=v\left(x_{i}\right)$ for every $i$. The following result replicates Lemma 5.4:

Lemma 5.9. For every $v \in H_{-\alpha}^{2}$ with $\alpha$ satisfying (5.1) and $\ell=0,1, \Pi_{h}$ satisfies the following properties:

$$
\begin{aligned}
\left|v-\Pi_{h} v\right|_{H^{\ell}(T)} & \leq C h_{T}^{2-\ell}|v|_{H^{2}(T)}, & & \forall T \in \mathcal{T}_{h} \\
\left|\Pi_{h} v\right|_{H_{-\alpha}^{1}(T)} & \leq C h_{T}^{-1}\|v\|_{L_{-\alpha}^{2}(T)}+|v|_{H_{-\alpha}^{1}(T)}+h_{T}|v|_{H_{-\alpha}^{2}(T)}, & & \forall T: T \cap\left\{x_{0}\right\} \neq \emptyset .
\end{aligned}
$$

Proof. Equation (5.8) is a well known result. For (5.9), the proof is the same than the one for Lemma 5.4, so we leave the details to the reader.

In (5.4), we took advantage of the fact that $u$ belongs to a Kondratiev type space, so $\nabla u \in L_{\alpha}^{2}$, but $u \in L_{\alpha-1}^{2}$. That is not true for $\varphi_{\beta}$ and thence we obtain the term $h_{T}^{-1}\|v\|_{L_{-\alpha}^{2}(T)}$.

In order to compensate the term $h_{T}^{-1}\|v\|_{L^{2}-\alpha(T)}$ in (5.9), we use that $\Pi_{h}$ is invariant over polynomials of degree 1. Let us define $P_{T}(v)$ the polynomial of degree 1 such that $\int_{T} D^{\alpha}\left(v(x)-P_{T}(v)(x)\right) \mathrm{d} x=0$, for every $|\alpha| \leq 1$. The following result is a natural consequence of Lemma 5.8:

Lemma 5.10. For $T \in \mathcal{T}_{h}$ such that $T \cap\left\{x_{0}\right\} \neq \emptyset$, taking $\alpha$ and $\beta$ as in Lemma 5.8, the following inequalities hold:

$$
\begin{aligned}
\left\|\nabla\left(v-P_{T}(v)\right)\right\|_{L_{-\alpha}^{2}(T)} & \leq C h_{T}^{1-\alpha+\beta}|v|_{H_{-\beta}^{2}(T)} \\
\left\|v-P_{T}(v)\right\|_{L_{-\alpha}^{2}(T)} & \leq C h_{T}^{2-\alpha+\beta}|v|_{H_{-\beta}^{2}(T)} .
\end{aligned}
$$

Proof. Equation (5.10) is given directly by Lemma 5.8. (5.11) follows applying first Lemma 5.8 with $\beta=\alpha$ and afterwards (5.10).

Now, we are finally able to prove our error estimate in $L_{\beta}^{2}$ :

Lemma 5.11. Let $\alpha$ satisfying (5.1), and $\beta \geq \alpha-1$. Taking $\mu \leq 1+\beta-\alpha$, we have:

$$
\left\|\nabla\left(w_{\beta}-\Pi_{h}\left(w_{\beta}\right)\right)\right\|_{L_{-\alpha}^{2}(\Omega)} \leq C h\left\|\Delta w_{\beta}\right\|_{L_{-\beta}^{2}(\Omega)} .
$$


Proof. We prove the result elementwise. For $T \in \mathcal{T}_{h}^{\text {far }}$ :

$$
\begin{aligned}
\left\|\nabla\left(w_{\beta}-\Pi_{h}\left(w_{\beta}\right)\right)\right\|_{L_{-\alpha}^{2}(T)} & \leq C r_{T}^{-\alpha}\left\|\nabla\left(w_{\beta}-\Pi_{h}\left(w_{\beta}\right)\right)\right\|_{L^{2}(T)} \\
& \leq C h_{T} r_{T}^{\beta-\alpha}\left|w_{\beta}\right|_{H_{-\beta}^{2}\left(S_{T}\right)} \leq C h r_{T}^{1-\mu+\beta-\alpha}\left|w_{\beta}\right|_{H_{-\beta}^{2}\left(S_{T}\right)} \leq C h\left|w_{\beta}\right|_{H_{-\beta}^{2}\left(S_{T}\right)}
\end{aligned}
$$

whereas, for $T \in \mathcal{T}_{h}^{\text {near }}$, we interpose $P_{T}=P_{T}\left(\varphi_{\beta}\right)$ :

$$
\left\|\nabla\left(w_{\beta}-\Pi_{h}\left(w_{\beta}\right)\right)\right\|_{L_{-\alpha}^{2}(T)} \leq\left\|\nabla\left(w_{\beta}-P_{T}\right)\right\|_{L_{-\alpha}^{2}(T)}+\left\|\nabla \Pi_{h}\left(w_{\beta}-P_{T}\right)\right\|_{L_{-\alpha}^{2}(T)}=\mathrm{I}+\mathrm{II} .
$$

Now, the second term can is bounded by (5.9):

$$
\mathrm{II} \leq h_{T}^{-1}\left\|w_{\beta}-P_{T}\right\|_{L_{-\alpha}^{2}(T)}+\left|w_{\beta}-P_{T}\right|_{H_{-\alpha}^{1}(T)}+h_{T}^{1+\beta-\alpha}|w|_{H_{-\beta}^{2}(T)} .
$$

In the last term we applied a slightly adapted version of (5.9), taking norm $L_{-\beta}^{2}$ only for the second order derivatives. Now, applying Lemma 5.10, we have:

$$
\| \nabla\left(w_{\beta}-\Pi_{h}\left(w_{\beta}\right) \|_{L_{-\alpha}^{2}(T)} \leq C h_{T}^{1-\alpha+\beta}\left|w_{\beta}\right|_{H_{-\beta}^{2}\left(S_{T}\right)} .\right.
$$

Summing up over all $T \in \mathcal{T}_{h}$ we obtain $\left\|w_{\beta}-\Pi_{h} w_{\beta}\right\|_{L_{-\alpha}^{2}(\Omega)} \leq C h\left|w_{\beta}\right|_{H_{-\beta}^{2}(\Omega)}$. Applying Theorem 3.1, the result follows.

Finally, we obtain optimal orders of convergence in $L_{\beta}^{2}$ :

Theorem 5.12. Let $u$ be the solution of (2.3) in $H_{\alpha}^{1}(\Omega), \eta<\alpha-1$ such that $u \in K_{\eta}^{2}(\Omega)$, and $\mu \leq \min \{1+$ $\beta-\alpha, \alpha-1-\eta$, then the following estimate holds:

$$
\left\|u-u_{h}\right\|_{L_{\beta}^{2}(\Omega)}^{2} \leq C h^{2}\|u\|_{K_{\eta}^{2}(\Omega)} .
$$

Proof. The result follows directly applying Lemma 5.11 in (5.7).

Remark 5.13. For $f=\delta_{x_{0}}$, we had (Rem. 5.7) condition $\mu<\alpha+1-\frac{n}{2}$. Combining this with $\mu \leq 1+\beta-\alpha$, we can choose $\alpha=\frac{\beta}{2}+\frac{n}{4}$ in order to maximize the range of values for $\mu$, obtaining that: If $\mu<1+\frac{\beta}{2}-\frac{n}{4}$, then:

$$
\left\|u-u_{h}\right\|_{L_{\beta}^{2}(\Omega)} \leq C h^{2} .
$$

In particular, taking $\beta=0$, we obtain an $L^{2}$ error $\sim O\left(h^{2}\right)$, if $\mu<1-\frac{n}{4}$.

Observe that Remark 5.13 in $n=2$ prescribes a graduation parameter $\mu<\frac{1}{2}$. In [4] the authors propose a graded mesh in $\mathbb{R}^{2}$ with parameter $\mu=\frac{1}{2}$, and prove the suboptimal rate of convergence:

$$
\left\|u-u_{h}\right\|_{L^{2}(\Omega)} \leq C h^{2}|\log (h)|^{\frac{3}{2}} .
$$

A similar result is obtained in [20]. Our numerical results are consistent with the ones exposed in [4], showing an order slightly worse than 2 for $\mu=\frac{1}{2}$. However, taking $\mu<\frac{1}{2}$ the optimal order is recovered (see Tabs. 1 and 2 in Sect. 6). Our result is also valid in $n=3$, giving a restriction $\mu<\frac{1}{4}$ in order to recover optimal rates of convergence. 


\section{NumERICAL EXPERIMENTS}

In this section we present our numerical results. We implemented a solver in Python, following closely the compact implementation proposed in [2].

We solve Problem (2.3) with $f=\delta_{x_{0}}, x_{0}$ the origin and $\Omega=B(0,1)$, where the exact solution is known.

The graded meshes for $n=2$ were obtained as in [4]: we built a quasi-uniform mesh of size $H$ with a set of points $Q=\{q\}$ and then scale this points taking: $p=q\|q\|^{\frac{1-\mu}{\mu}}$. In this way, for each $H$ we have a collection of meshes with different graduations, but the same number of nodes. Figure 1 shows three meshes in $\mathbb{R}^{2}$ : uniform, graded with $\mu=0.5$ and graded with $\mu=0.3$.

Tables 1 and 2 show results for $n=2$ on meshes graded with $\mu=0.3,0.4,0.5$ and $\mu=1$ (quasi-uniform).
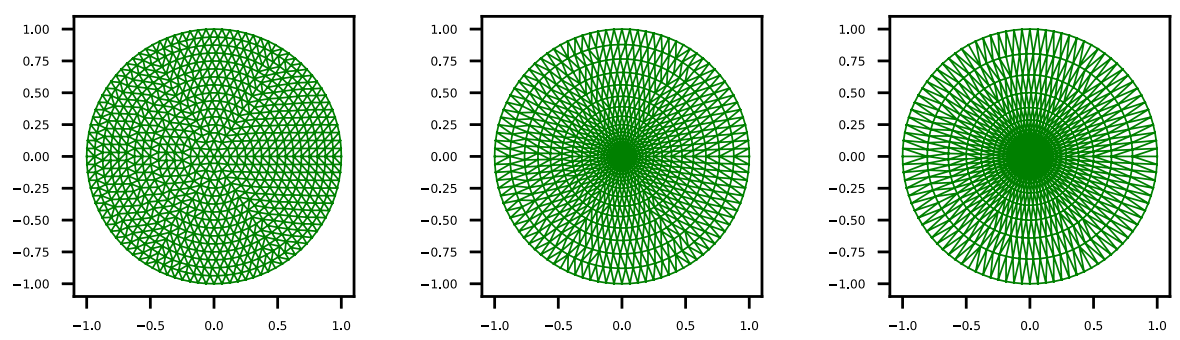

FiguRE 1. Uniform mesh (left), graded mesh with $\mu=0.5$ (center) and $\mu=0.3$ (right). $N=847$.

TABLE 1. Error in $L^{2}, L_{\beta_{0}}^{2}$ and $L_{\beta_{1}}^{2}\left(\beta_{0}=-0.2, \beta_{1}=0.4\right)$, and in $H_{\alpha_{0}}^{1}\left(\alpha_{0}=0.4\right)$.

\begin{tabular}{|c|c|c|c|c|c|c|c|c|c|}
\hline \multirow[b]{2}{*}{$h$} & \multirow[b]{2}{*}{$N$} & \multicolumn{4}{|c|}{$\mu=0.3$} & \multicolumn{4}{|c|}{$\mu=0.4$} \\
\hline & & $\begin{array}{l}L^{2} \\
\times 10^{-5}\end{array}$ & $\begin{array}{l}L_{\beta_{0}}^{2} \\
\times 10^{-5}\end{array}$ & $\begin{array}{l}L_{\beta_{1}}^{2} \\
\times 10^{-5}\end{array}$ & $\begin{array}{l}H_{\alpha_{0}}^{1} \\
\times 10^{-3}\end{array}$ & $\begin{array}{l}L^{2} \\
\times 10^{-5}\end{array}$ & $\begin{array}{l}L_{\beta_{0}}^{2} \\
\times 10^{-5}\end{array}$ & $\begin{array}{l}L_{\beta_{1}}^{2} \\
\times 10^{-5}\end{array}$ & $\begin{array}{l}H_{\alpha_{0}}^{1} \\
\times 10^{-3}\end{array}$ \\
\hline $2^{-5}$ & 3334 & 22.36 & 32.39 & 17.75 & 13.39 & 14.68 & 29.13 & 10.23 & 16.65 \\
\hline $2^{-6}$ & 13102 & 5.601 & 8.201 & 4.443 & 5.306 & 3.703 & 8.008 & 2.558 & 8.29 \\
\hline $2^{-7}$ & 51938 & 1.4 & 2.061 & 1.110 & 2.105 & 0.93 & 2.169 & 0.639 & 4.137 \\
\hline $2^{-8}$ & 206819 & 0.35 & 0.516 & 0.277 & 0.836 & 0.233 & 0.58 & 0.159 & 2.067 \\
\hline $2^{-9}$ & 825671 & 0.087 & 0.129 & 0.069 & 0.332 & 0.058 & 0.153 & 0.04 & 1.03 \\
\hline $2^{-10}$ & 3297924 & 0.022 & 0.032 & 0.017 & 0.133 & 0.015 & 0.04 & 0.01 & 0.517 \\
\hline & e.o.c & 2.008 & 2.003 & 2.008 & 1.336 & 2.003 & 1.905 & 2.007 & 1.007 \\
\hline
\end{tabular}

TABLE 2. Error in $L^{2}, L_{\beta_{0}}^{2}$ and $L_{\beta_{1}}^{2}\left(\beta_{0}=-0.2, \beta_{1}=0.4\right)$, and in $H_{\alpha_{0}}^{1}\left(\alpha_{0}=0.4\right)$.

\begin{tabular}{|c|c|c|c|c|c|c|c|c|c|}
\hline \multirow[b]{2}{*}{$h$} & \multirow[b]{2}{*}{$N$} & \multicolumn{4}{|c|}{$\mu=0.5$} & \multicolumn{4}{|c|}{$\mu=1$} \\
\hline & & $\begin{array}{l}L^{2} \\
\times 10^{-5}\end{array}$ & $\begin{array}{l}L_{\beta_{0}}^{2} \\
\times 10^{-5}\end{array}$ & $\begin{array}{l}L_{\beta_{1}}^{2} \\
\times 10^{-5}\end{array}$ & $\begin{array}{l}H_{\alpha_{0}}^{1} \\
\times 10^{-3}\end{array}$ & $\begin{array}{l}L^{2} \\
\times 10^{-4}\end{array}$ & $\begin{array}{l}L_{\beta_{0}}^{2} \\
\times 10^{-4}\end{array}$ & $\begin{array}{l}L_{\beta_{1}}^{2} \\
\times 10^{-5}\end{array}$ & $\begin{array}{l}H_{\alpha_{0}}^{1} \\
\times 10^{-2} \\
\end{array}$ \\
\hline $2^{-5}$ & 3334 & 13.62 & 35.82 & 6.933 & 23.06 & 5.557 & 12.07 & 1.424 & 7.263 \\
\hline $2^{-6}$ & 13102 & 3.675 & 11.92 & 1.734 & 13.22 & 2.779 & 6.932 & 5.41 & 5.504 \\
\hline $2^{-7}$ & 51938 & 0.982 & 3.968 & 0.434 & 7.59 & 1.389 & 3.981 & 2.053 & 4.171 \\
\hline $2^{-8}$ & 206819 & 0.26 & 1.314 & 0.108 & 4.359 & 0.695 & 2.287 & 0.778 & 3.161 \\
\hline $2^{-9}$ & 825671 & 0.068 & 0.423 & 0.027 & 2.499 & 0.353 & 1.339 & 0.297 & 2.4 \\
\hline $2^{-10}$ & 3297924 & 0.018 & 0.014 & 0.007 & 1.438 & 0.174 & 0.754 & 0.112 & 1.816 \\
\hline & e.o.c & 1.922 & 1.6 & 2.005 & 0.804 & 1.005 & 0.804 & 1.405 & 0.402 \\
\hline
\end{tabular}


TABLE 3. Error in $L^{2}, L_{\beta_{0}}^{2}$ for $\beta_{0}=0.4$, and in $H_{\alpha_{0}}^{1}$ with $\alpha_{0}=0.75$, and estimated order of convergence, for different graduations.

\begin{tabular}{lllllllll}
\hline \hline & & \multicolumn{3}{c}{$\mu=0.2$} & \multicolumn{4}{c}{$\mu=0.25$} \\
\cline { 3 - 9 }$h$ & $N$ & $\begin{array}{l}L^{2} \\
\times 10^{-4}\end{array}$ & $\begin{array}{l}L_{\beta_{0}}^{2} \\
\times 10^{-4}\end{array}$ & $\begin{array}{l}H_{\alpha_{0}}^{1} \\
\times 10^{-3}\end{array}$ & $N$ & $\begin{array}{l}L^{2} \\
\times 10^{-4}\end{array}$ & $\begin{array}{l}L_{\beta_{0}}^{2} \\
\times 10^{-4}\end{array}$ & \begin{tabular}{l}
$H_{\alpha_{0}}^{1} \times 10^{-3}$ \\
\hline $2^{-3}$
\end{tabular} \\
\cline { 3 - 9 } & 18028 & 12.72 & 4.896 & 21.34 & 14070 & 16.46 & 5.356 & 6.46 \\
$2^{-3.5}$ & 45592 & 7.238 & 2.643 & 14.92 & 33136 & 10.09 & 2.938 & 6.46 \\
$2^{-4}$ & 110540 & 3.859 & 1.387 & 9.935 & 91798 & 5.55 & 1.515 & 4.74 \\
$2^{-4.5}$ & 298737 & 1.994 & 0.706 & 6.552 & 237721 & 3.101 & 0.994 & 3.43 \\
$2^{-5}$ & 807040 & 1.03 & 0.363 & 4.339 & 646643 & 1.652 & 0.413 & 2.40 \\
$2^{-5.5}$ & 2161204 & 0.526 & 0.187 & 2.866 & 1813404 & 0.893 & 0.215 & 2.40 \\
\hline \multicolumn{2}{c}{ e.o.c } & 1.996 & 2.072 & 1.258 & & 1.799 & 2.11 & 1.04 \\
\hline
\end{tabular}

Norms in $L^{2}, L_{\beta_{0}}^{2}, L_{\beta_{1}}^{2}$ and $H_{\alpha_{0}}^{1}$ are considered, where $\beta_{0}=-0.2, \beta_{1}=0.4$ and $\alpha_{0}=0.4$. The estimated order of convergence (e.o.c.) is computed with respect to $\frac{1}{N^{2}}$ (recall Rem. 4.1). The value of $h$ used for building the meshes is reported only for informative purposes. The results are consistent with our theoretical predictions. Indeed, we observe an e.o.c. of 2 in $L^{2}$ for $\mu=0.3$ and $\mu=0.4$, which deteriorates slightly for $\mu=0.5$ and is reduced to 1 for $\mu=1$, as proved in [8]. On the other hand, the norm $L_{\beta_{0}}^{2}$ preserves the optimal order of convergence for $\mu=0.3$ as expected (since $\mu<\frac{1}{2}+\frac{\beta_{0}}{2}$ ), but impoverishes it for $\mu=0.4$. In $L_{\beta_{1}}^{2}$, the optimal order of convergence is also obtained for $\mu=0.5$. According to Remark 5.7, we need $\mu<\alpha_{0}$ in order to obtain order 1 in $H_{\alpha_{0}}^{1}$, which is verified for $\mu=0.3$. Larger values of $\mu$ deteriorates the order of convergence, which is $\sim \frac{\alpha_{0}}{\mu}$, in agreement with Corollary 5.6 .

For $n=3$ meshes graded by scaling a quasi-uniform mesh did not work as well as for $n=2$. This is probably due to the fact that the number of points near the singularity is unnecessarily large. Hence, we build meshes that are graded by construction: we define a set of radii, graded towards zero. For each radium $r$, we take the element-size $h(r) \sim h r^{1-\mu}$ and produce a list of points at relative distance $\sim h(r)$ on the sphere of radious $r$. Joining the points on all the spheres with the origin, we obtain the set of vertices of our triangulation. The only setback of this strategy is that meshes build from the same mesh parameter $h$ but with different grading parameter $\mu$ have different number of points. However, recall that Remark 4.1 guarantees that the number of elements remains $N \sim h^{-3}$ for every $\mu>\mu_{0}$. Given this particular distribution of points, a naïve application of direct Delaunay algorithms produces very low-quality tetrahedra, that do not satisfy the grading restrinctions, nor the isotropic nature of the mesh. Hence, we used TetGen [29], which allows quality improvements on the meshes, setting, for example, the ratio between the diameter and the length of the edges (we set it at 1.5), lower bounds on the internal dihedral angles (we set it at 10 degrees), etc.

Tables 3 and 4 shows results for $n=3$ on meshes graded with $\mu=0.2,0.25$ and 0.5 in $L_{\beta_{0}}^{2}$ and $H_{\alpha_{0}}^{1}$, with $\beta_{0}=0.4, \alpha_{0}=0.75$.

Again, we are recovering the predicted order of convergence. The order $\sim 2$ for $\mu=0.2$ is reduced to $\sim 1.8$ for the critical value $\mu=0.25$ and to 1 for $\mu=0.5$. These results are naturally improved for $\beta=0.4$, where we have order $\sim 2$ for $\mu=0.25$, also. In $H_{\alpha}^{1}$ an order of 1.25 is obtained for $\mu=0.2$. This (too good) result is due to the fact that our domain has radious 1 , so the main contribution to the error is given by the elements near the singularity, where an estimate $h^{\frac{\alpha-1-\eta}{\mu}}$ holds. Taking $\alpha=0.75, \mu=0.2$ and $\eta>-0.5$, we obtain an exponent $\sim \frac{0.25}{0,2}=1.25$. The other cases are given similarly by Corollary 5.6 .

In conclusion, our experimental results are consistent with our theoretical predictions.

Acknowledgements. I thank Ricardo Durán for suggesting the use of reference [25] for obtaining Theorem 3.1. I also want to thank the anonymous referees for their valuable comments, that have helped to improve the paper. 
TABLE 4. Error in $L^{2}, L_{\beta_{0}}^{2}$ for $\beta_{0}=0.4$, and in $H_{\alpha_{0}}^{1}$ with $\alpha_{0}=0.75$, and estimated order of convergence, for different graduations.

\begin{tabular}{lllllllll}
\hline \hline & & \multicolumn{3}{c}{$\mu=0.5$} & \multicolumn{5}{c}{$\mu=1$} \\
\cline { 3 - 9 }$h$ & $N$ & $\begin{array}{l}L^{2} \\
\times 10^{-3}\end{array}$ & $\begin{array}{l}L_{\beta_{0}}^{2} \\
\times 10^{-4}\end{array}$ & $\begin{array}{l}H_{\alpha_{0}}^{1} \\
\times 10^{-2}\end{array}$ & $N$ & $\begin{array}{l}L^{2} \\
\times 10^{-3}\end{array}$ & $\begin{array}{l}L_{\beta_{0}}^{2} \\
\times 10^{-3}\end{array}$ & \begin{tabular}{l}
$H_{\alpha_{0}}^{1} \times 10^{-1}$ \\
\hline $2^{-3}$
\end{tabular} \\
\cline { 3 - 9 } & 6914 & 3.56 & 9.912 & 8.735 & 2504 & 8.286 & 3.562 & 1.57 \\
$2^{-3.5}$ & 15698 & 2.645 & 6.143 & 7.523 & 8076 & 6.768 & 2.478 & 1.418 \\
$2^{-4}$ & 43506 & 1.866 & 3.424 & 6.265 & 18818 & 5.861 & 1.913 & 1.319 \\
$2^{-4.5}$ & 112723 & 1.414 & 2.014 & 5.31 & 55182 & 4.889 & 1.38 & 1.205 \\
$2^{-5}$ & 307073 & 1.017 & 1.142 & 4.467 & 146278 & 4.145 & 1.026 & 1.109 \\
$2^{-5.5}$ & 854491 & 0.719 & 0.633 & 3.75 & 431554 & 3.46 & 0.741 & 1.013 \\
\hline \multicolumn{2}{c}{ e.o.c } & 0.998 & 1.714 & 0.527 & & 0.508 & 0.915 & 0.255 \\
\hline
\end{tabular}

\section{REFERENCES}

[1] J.P. Agnelli, E.M. Garau and P. Morin, A posteriori error estimates for elliptic problems with dirac measure terms in weighted spaces. ESAIM: M2AN 48 (2014) 1557-1581.

[2] J. Alberty and S.A. Funken, Remarks around 50 lines of MATLAB: short finite element implementation. Numer. Algorithms 20 (1999) 117-137.

[3] T. Apel, A.-M. Sändig and J.R. Whiteman, Graded mesh refinement and error estimates for finite element solutions of elliptic boundary value problems in non-smooth domains. Math. Methods Appl. Sci. 19 (1996) 63-85.

[4] T. Apel, O. Benedix, D. Sirch and B. Vexler, A priori mesh grading for an elliptic problem with Dirac right-hand side. SIAM J. Numer. Anal. 49 (2011) 992-1005.

[5] R. Araya, E. Behrens and R. Rodriguez, A posteriori error estimates for elliptic problems with Dirac delta source terms. Numer. Math. 196 (2007) 2800-2812.

[6] I. Babuska, Error-bounds for finite element method. Numer. Math. 16 (1971) 322-333.

[7] S.C. Brenner and L.R. Scott, The mathematical theory of finite element methods, 3rd edition. In: Vol. 15 of Texts in Applied Mathematics. Springer, New York (2008).

[8] E. Casas, $L^{2}$ Estimates for the finite element method for the Dirichlet problem with singular data. Numer. Math. 47 (1985) 627-632.

[9] E. Cejas and R.G. Durán, Weighted a priori estimates for elliptic equations. Studia Math. 243 (2018) 13-24.

[10] C. D'Angelo, Finite element approximation of elliptic problems with Dirac measure terms in weighted spaces: applications to one- and three-dimensional coupled problems. SIAM J. Numer. Anal. 50 (2012) 194-215.

[11] I. Drelichman and R. Durán, Improved Poincaré inequalities with weights. J. Math. Anal. App. 347 (2008) $286-293$.

[12] I. Drelichman, R. Durán and I. Ojea, A weighted setting for the Poisson problem with singular sources. SIAM J. Numer. Anal. 58 (2019) 590-60.

[13] J. Duoandikoetxea, Forty Years of Muckenhoupt Weights, Function Spaces and Inequalities. Charles University and Academy of Sciences, Prague (2013).

[14] R. Durán and F. López García, Solutions of the divergence and analysis of the Stokes equation in planar Hölder- $\alpha$ domains. Math. Models Methods. Appl. Sci. 20 (2010) 95-120.

[15] D. Gilbarg and N.S. Trudinger, Elliptic partial differential equations of second order, 2nd edition. In: Vol. 224 of Grundlehren der Mathematischen Wissenschaften [Fundamental Principles of Mathematical Sciences]. Springer, Berlin-Heidelberg (1983).

[16] P. Grisvard, Elliptic Problems in Nonsmooth Domains. Pitman Publishing Inc., Marshfield, MA (1985).

[17] J. Guzmán, D. Leykekhman, J. Rossmann and A.H. Schatz, Hölder estimates for Green's functions on convex polyhedral domains and their applications to finite element methods. Numer. Math. 112 (2009) 221-243.

[18] L.I. Hedberg, On certain convolution inequalities. Proc. Amer. Math. Soc. 36 (1972) 505-510.

[19] D. Jerison and C.E. Kenig, The inhomogeneous Dirichlet problem in Lipschitz domains. J. Funct. Anal. 130 (1995) $161-219$.

[20] T. Köppl and B. Wohlmuth, Optimal a priori error estimates for an eliptic problem with Dirac right-hand side. SIAM J. Num. An. 52 (2014) 1753-1769.

[21] H. Li, The $W_{p}^{1}$ stability of the Ritz projection on graded meshes. Math. Comp. 86 (2017) 49-74.

[22] V.G. Maz'ya and J. Rossmann, Weighted Lp estimates of solutions to boundary value problems for second order elliptic systems in polyhedral domains. Z. Angew. Math. Mech. 83 (2003) 435-467.

[23] V.G. Maz'ya and J. Rossmann, Elliptic equations in polyhedral domains. In: Vol. 162 of Mathematical Surveys and Monographs. American Mathematical Society, Providence, RI (2010).

[24] R. Nochetto, E. Otárona and A. Salgado, Piecewise polynomial interpolation in Muckenhoupt weighted Sobolev spaces and applications. Numer. Math. 132 (2016) 85-130. 
[25] E. Otárola and A. Salgado, The Poisson and Stokes problems on weighted spaces in Lipschitz domains and under singular forcing. J. Math. Anal. App. 471 (2018) 599.

[26] J.V. Pellegrotti, E. Cortés, M.D. Bordenave, M. Caldarola, M.P. Kreuzer, A.D. Sánchez, et al., Plasmonic photothermal fluorescence modulation for homogeneous biosensing. ACSSensors 1 (2016) 1351-1357.

[27] E. Sawyer and R.L. Wheeden, Weighted inequalities or fractional integrals on euclidean and homogeneous spaces. Am. J. Math. 114 (1992) 813-874.

[28] L.R. Scott, Finite element convergence for singular data. Numer. Math. 21 (1973) 317-327.

[29] H. Si, TetGen, a Delaunay-based quatily tetrahedral mesh generator. ACM Trans. Math. Softw. 41 (2015) 1-11. 